\title{
TV/Series
}

Hors séries 1 | 2016

Lost: (re)garder l'île

\section{The Leftovers, the Lost fever}

Double frénésie en séries pour Damon Lindelof

\section{Guillaume Dulong}

\section{(2) OpenEdition}

\section{Journals}

Édition électronique

URL : http://journals.openedition.org/tvseries/4371

DOI : $10.4000 /$ tvseries. 4371

ISSN : 2266-0909

Éditeur

GRIC - Groupe de recherche Identités et Cultures

Référence électronique

Guillaume Dulong, "The Leftovers, the Lost fever », TV/Series [En ligne], Hors séries 1 | 2016, mis en ligne le 30 juin 2020, consulté le 02 juillet 2020. URL : http://journals.openedition.org/tvseries/4371 ; DOI : https://doi.org/10.4000/tvseries.4371

Ce document a été généré automatiquement le 2 juillet 2020

\section{(c) $(1)$}

TV/Series est mis à disposition selon les termes de la licence Creative Commons Attribution - Pas d'Utilisation Commerciale - Pas de Modification 4.0 International. 


\title{
The Leftovers, the Lost fever
}

\author{
Double frénésie en séries pour Damon Lindelof
}

\section{Guillaume Dulong}

\begin{abstract}
Le 22 septembre, aujourd'hui je m'en fous.
Et c'est triste de n'être plus triste sans vous ${ }^{1}$. Comme Arthur le dit de son île: « Je préfère faire comme si elle n'avait pas existé. C'est pourquoi, jusqu'au moment où l'on n'en verra plus rien, il

vaut mieux que tu ne regardes pas dans cette direction. Quant à toi, préviens-moi à ce moment-
\end{abstract}

là²."

1 Le 4 juin 2017, Damon Lindelof, achevait, sur HBO, une série intitulée The Leftovers. Avec le huitième épisode, "The Book of Nora ", se clôturait la troisième saison ainsi que cette histoire initiée en 2013. Saluée par la critique, cette fin tire-t-elle les leçons du tollé que suscita, sept ans auparavant, la fin de la précédente série du showrunner, Lost, diffusée sur $\mathrm{ABC}$ de 2004 à 2010 ? Achever The Leftovers fut-il, pour Lindelof et les téléspectateurs ayant suivi les deux shows, une manière d'achever l'achèvement de Lost ? Bien entendu, cela implique que chacune de ces séries n'est pas qu'un récit clos. Ainsi ce qu'elles racontent fait-il sens littéralement (en regard de leurs univers poétiques de référence), allégoriquement (dans le rapport desdits univers avec des références au monde ordinaire), mais aussi en prenant en compte une dimension intertextuelle. Cette dernière interroge la pratique voire la métaphysique de l'acte de raconter et de recevoir un récit. Dans ce cas, l'intrigue de The Leftovers parle un monde qui n'est pas le nôtre mais qui «ressemble» au nôtre. Donc, l'intrigue parle aussi de cette relation spéciale de la fiction télévisuelle au réel qui, à la fois, le rend visible (vision) en le mettant à distance (télé), tout en s'inscrivant elle-même, directement ou indirectement, dans une histoire composée de récits. Comme Philip Jeffries (David Bowie) clamant haut et fort qu'il ne parlera pas de Judy dans Twin Peaks: Fire Walk with me (réal. David Lynch, 1992), pour mieux se contredire dans les Missing Pieces, annoncer qu'on ne traitera pas de Lost en se donnant pour tâche d'analyser The Leftovers, serait s'imposer une double contrainte. En effet, dans les deux récits nous retrouvons une certaine veine fantastique, ou affiliée, pour peu que nous reprenions la définition du genre proposée 
par Todorov ${ }^{3}$. Les intrigues nous rapportent les affres des membres d'une communauté à la fois unis et divisés par une catastrophe : ici le crash d'un avion sur une île située dans le Pacifique Sud, le 22 Septembre 2002, là la disparition soudaine de $2 \%$ de la population mondiale, le 14 Octobre 2011. De cet incident originel, chacun se demande s'il répond à un ordre naturel explicable, un plan surnaturel à craindre ou à aimer, ou à un insondable désordre ${ }^{4}$.

2 Ici et là, nous avons des hommes de sciences rationalistes et pragmatiques pour qui il existe une frontière claire et distincte entre causalité et contiguïté, entre croyance et science et une subordination de celle-là à celle-ci. Là et ici, nous retrouvons des hommes de foi, religieux et initiés, pour qui la frontière est moins nette, et pour qui le savoir doit, sinon se soumettre, pour le moins reconnaître les limites de son domaine de validité. Quant au reste, let the mystery be... L'enjeu de cette interrogation intradiégétique a une résonance métadiégétique: l'échange entre l'écriture et la réception qu'institue le récit n'est-il régi que par des règles objectives? Quelle part joue notre désir dans le crédit accordé à l'unité d'une intrigue comme promesse de résolution d'un incident initial ? Y a-t-il une frontière entre une explication objective et logique de ces histoires et une compréhension subjective?

Dans le cadre de cette réflexion herméneutique, les homologies de Lost et The Leftovers, nous amènent à concevoir l'apocalyptique récit de cette dernière série comme étant une antithèse parodique de Lost radicalisant les tensions contenues et suscitées par son intrigue. Nous montrerons que la série diffusée sur $\mathrm{HBO}$ est le show diffusé sur $\mathrm{ABC}$ en régime de double frénésie, dans le sens apocalyptique que lui prête Girard. Ce dernier s'inspire d'une expression de Bergson dans Les Deux sources de la morale et de la religion, qu'il subvertit. Bergson y voyait une loi de l'histoire oscillant entre des phases de progrès matériels et techniques et des phases de progrès spirituels. Girard entend plutôt cette loi historique comme une montée aux extrêmes de la réciprocité violente. Le cours de cet extrémisme est non seulement de moins en moins bien canalisé et différé par les divertissements et substituts sacrificiels, sans inflation considérable, mais de surcroît ces détournements en viennent même à en toujours davantage confirmer le péril. L'histoire devient donc un compte à rebours (doomsday clock). Seul espoir, sa progression révélant le mensonge et l'inefficace de toute entreprise de substitution sacrificielle, est corrélativement rebroussement vers l'origine, la genèse, « rapprochement accéléré du début et de la fin des temps ${ }^{5}$. » Cette loi de double frénésie est donc apocalyptique dans les deux sens du terme: l'histoire est catastrophe et révélation.

4 The Leftovers est, en ce sens une parodie extrême, une relecture malade de Lost, The Lost fever, s'apparentant à un Lost raconté par le monstre de fumée, l'Homme sans nom (Titus Welliver), le frère ennemi de Jacob (Mark Pellegrino). C'est l'histoire vue par celui qui veut fuir à tout prix, par celui qui veut détruire enfin l'île et, avec elle, l'univers narratif, bouc émissaire de sa frustration et échappatoire de son ressentiment par rapport au monde commun. Notre hypothèse serait alors que si un tel démiurge devait faire un récit, il serait un narrateur peu digne de confiance. Or dans The Leftovers, dans la troisième saison, un personnage s'autoproclame Dieu, il est irresponsable, assassin et se nomme David Burton (Bill Camp). On l'aperçoit dans l'autre monde, accompagnant Kevin (Justin Theroux), une corde à la main sur le pont. Il lui chuchote qu'il est l'homme le plus puissant du monde dans l'épisode "International Assassin " (S02E08). Puis, dans l'épisode " The most powerful man in the world and his identical 
twin brother » (S03E07), il le guide jusqu'à la fin du monde. Dieu rencontre aussi Matt (Christopher Eccleston) dans le monde réel de la diégèse, dans l'épisode «It's a Matt Matt World » (S03E05).

5 Cette hypothèse d'une série télévisée projetée par un mauvais œil se jouant de la souffrance de ses personnages en se focalisant sur des bêtes noires, nous amènera à examiner la prégnance du thème du bouc émissaire dans le finale la série « The Book of Nora ». Ce motif était préfiguré sous une forme rituelle à Jarden dans l'épisode «Lens » (S02E06) où une chèvre était égorgée tandis que Nora (Carrie Coon) était accusée d'être l'incarnation d'Azraël. Dans le dernier épisode, Nora se substitue à l'animal avant de révéler à Kevin le mystère sur lequel s'ouvre la série : les $2 \%$ de la population mondiale ont disparu en raison d'ondes qui font que, réciproquement, les leftovers (auxquels nous nous identifiions depuis le début) ont disparu pour eux. Le mystère sera alors de savoir si Kevin veut bien croire que Nora dit la vérité car nous ne verrons jamais cet autre côté des ondes. Précédant le dénouement et la reconnaissance finale, comme acmé de l'épisode, le motif du bouc émissaire ne prend-il pas ainsi une dimension intertextuelle et métadiégétique ? Ce rituel ne fait-il pas référence à ce que fait la fiction télévisuelle? La série montrerait ainsi que la fiction télévisuelle tend généralement à nous tromper et nous divertir du réel en un spectacle qui nous vend une légende officielle cachant des victimes officieuses offertes en sacrifice à notre regard. Ce qui impliquerait alors que le point de vue même de The Leftovers se ferait à travers une lentille persécutrice et mystifiante se focalisant sur Nora, personnage en révélant les anamorphoses. Mais si les péripéties de The Leftovers sont les simulacres de cet écran de fumée qu'était le double de Jacob, est-ce à dire que tout est perdu dans The Leftovers? Que la série est l'apocalypse de Lost?

6 Cette dernière interrogation guidera notre étude de la structure de la saison 3 et en particulier de l'opposition des premiers et derniers épisodes, "The Book of Kevin » et "The Book of Nora ». Si, en regard de Lost, les deux premières saisons de The Leftovers sont une vision déchue du monde, la troisième déjoue les règles de composition déployées dans les précédentes, dans son traitement des génériques et du nombre d'épisodes, 10 dans celles-ci et 8 dans celle-là. La dernière saison se construit en confrontant deux livres. Sont ainsi prescrites deux manières antagonistes de lire et regarder la série : le Sudden Departure de Kevin et le Sudden Departure de Nora. Le monde en dystopie résultant d'une déformation mystificatrice et mensongère du show télévisé serait le fait de la version de Kevin. Cette déformation est dévoilée par la version de Nora qui entrouvre la perspective d'une fonction moins désespérée de l'art du récit et de la télévision en renouant in extremis avec Lost, via la conversion d'un tiers. Ce tiers est le narrateur délégué caché du récit, Matthew Jamison, se révélant dans l'épisode «It's a Matt, Matt World ». Cet épisode s'ouvre sur la destruction d'une île du Pacifique Sud et se referme sur la mort de Dieu dévoré aux aurores par un Lion au son off du Que c'est triste Venise d'Aznavour. Sa structure serait-elle une antithèse parodique de l'épisode de Lost «The Incident " (S05E17) se terminant sur l'explosion d'une bombe $\mathrm{H}$ sur l'île et sur la mort de Jacob? Dès lors, en reprenant la définition de la parodie que propose Giorgio Agamben, ne pourrions-nous pas espérer que "It's a Matt Matt world » soit porteur d'une signification messianique, annonçant l'avènement de Jacob et un autre régime d'image dont est porteur le livre de Nora par opposition à celui de Kevin? 


\section{The Lost Fever}

7 Nombre de critiques ont salué en The Leftovers, et en l'épisode "The Book of Nora ", une amende honorable de Damon Lindelof par rapport à la fin de Lost. Une fin plus mûre, moins naïve que celle de la série d'ABC, maturité confirmée par le choix de HBO comme prestigieux diffuseur pour une série plus sombre réalisée par des cinéastes (Peter Berg, Mimi Leder...). Son nombre d'épisodes, plus ramassé, parait en outre au reproche économique de perte de temps formulé à l'encontre de Lost. La tutelle de l'adaptation littéraire et la présence de l'auteur, Tom Perotta, étaient gages de " sérieux » d'un récit sachant où il va. Lindelof alimentait cette interprétation à longueur d'entretiens, se déclarant traumatisé d'avoir fait l'objet d'un lynchage numérique par certains fans de la première heure, le soir de la diffusion du double épisode de Lost, "The End », le 23 mai 2010. Sorti grandi de cette expérience, il put la sublimer dans l'écriture de The Leftovers et de sa fin, qualifiées d'accomplissements créatifs.

8 En aucun cas, néanmoins, il ne renie Lost, même et surtout lorsqu'il confie qu'il a appris à faire davantage confiance aux autres scénaristes, à Tom Perotta et à la réalisatrice Mimi Leder, alors qu'il relisait systématiquement les scripts de ses partenaires pendant la production de la première série. Le 20 juin 2017, il confie au journaliste Adam Chitwood : "Very often I can watch The Leftovers and have that same feeling because I've been able to let go, it's not my own ego $^{6}$. ". Toutefois il n'en persiste pas moins, dans ce cas, à utiliser une formule récurrente dans Lost, à savoir let go. Comme si déléguer son écriture et sacrifier son ego n'était finalement que le résultat d'une compréhension achevée du show de $\mathrm{ABC}$. Ce qui nous amène à conjecturer qu'il comprit très tôt, durant l'écriture des épisodes de Lost que la violence de la réaction de certains spectateurs serait inévitable. Les plus radicaux d'entre eux attendaient une explication définitive à toutes les énigmes soulevées par l'histoire et donc en finir avec son mystère central pour pouvoir enfin en parler au passé et ressaisir les péripéties en une totalité ordonnée dans une lecture rétrospective et achevée. En proposant de faire du show une expérience pré-mortem communautaire voire un rêve partagé, les auteurs se virent accusés de dévaluer brutalement, de brader, la résolution promise du mystère de l'île dans lequel tant de fans avaient investi en spéculations herméneutiques. D'ailleurs, dès la fin de la saison 5 avec le meurtre de Jacob puis, avec la saison 6, ses flashsideways résolvant l'intrigue en un appel à candidats de téléréalité, on sentait un air de profanation dans le sens qu'Agamben donne à ce terme, c'est-à-dire que Cuse et Lindelof rendaient à l'usage commun ce que le cours du récit avait consacré ce qui, en le showrunner, a le plus souffert de la réaction des spectateurs les plus véhéments, voire ingrats, fut la part d'ego qu'il mit dans son écriture et la réception idéale qu'il en attendait. Lui-même pensait peut-être obtenir de son public une reconnaissance sur le mode d'un retour sur investissement. À cet égard la fin de The Leftovers serait plus satisfaisante. D'une part, la dépense en temps d'écriture et de réception est moindre. D'autre part, elle est plus maligne que la naïve fin de Lost : nous savons ce qu'il advint des $2 \%$ mais le personnage de Nora pouvant tout aussi bien mentir, le spectateur garderait la liberté de ne pas croire.

9 The Leftovers corrige-t-il cependant le tir afin de rectifier l'impression d'inachèvement lostienne et son malentendu afin d'être politiquement correct? The Leftovers achève-t-il Lost pour rassurer ses thuriféraires et rouvrir les possibilités d'un récit fini dans une répétition (un « remember ») nostalgique ou bien cherche-t-il surtout à répondre aux 
attentes des détracteurs de la première série ? Dans The Leftovers, le troisième côté du miroir, Sarah Hatchuel et Pacôme Thiellement ne considèrent pas que le show n'est qu'un ajustement aux canons de réception des foules numériques et un récit plus mûr car plus réaliste, entropique et apocalyptique sur l'incommunicabilité entre les êtres. Cette nouvelle histoire est une antithèse, une version dévoyée de Lost. L'univers narratif de la fiction de $\mathrm{HBO}$ en regard de celle de celle d'ABC, pourrait bien être celui de l'homme en noir, le jumeau du démiurge de Lost, Jacob, qui veut en finir avec l'île ${ }^{8}$. Les auteurs font remarquer que là où The Tempest de Shakespeare orientait l'écriture de la première série, la seconde lui substituait l'une des pièces les plus gores du même dramaturge, tragédie à la limite du grand guignol, Titus Andronicus, notamment dans l'épisode " No Room in the Inn » (S02E05) ${ }^{9}$. Nous ne pouvons qu'abonder en ce sens, au moins concernant les deux premières saisons de The Leftovers. Cela paraît d'autant plus confirmé par ce que nous signalait Sarah Hatchuel, à savoir que le prénom de l'acteur interprétant le jumeau de Jacob est Titus. Or sachant que Lost, comme The Leftovers, intègrent une réflexion parodique sur les noms et prénoms des interprètes des personnages dans l'écriture du show, il est fort probable que The Leftovers soit le monde de Titus.

Qu'est ce monde? Exposé. Dans l'épisode Accross the Sea (S06E15), l'Homme sans Nom est défini par Mère (Allison Janney) comme spécial. Ce terme est suffisamment répété dans la série, en particulier par Locke (Terry O'Quinn) dont l'Homme sans nom prend l'apparence pour que nous en examinions le sens profond. Être spécial, c'est à la fois être spécifique (voire spécialissime) et spéculaire, c'est être une image, c'est-à-dire un être qui n'existe que comme accident de..., un être qui advient dans quelque chose d'autre, un incident dans le vocabulaire lostéen. Or comme l'écrit Agamben :

11 L'image est un être dont l'essence est d'être une espèce, une visibilité ou une apparence. Spécial, l'être dont l'essence coïncide avec le spectacle qu'il donne à voir, avec sa propre espèce.

12 L'être spécial est absolument non substantiel. Il n'a pas de lieu propre, mais il échoit à un sujet, et il s'y trouve comme un habitus ou comme un mode d'être, comme l'image dans le miroir ${ }^{10}$.

13 C'est par l'action de la lumière et pour nos yeux qu'apparaît l'image, de même qu'apparaissent les entités spéciales identifiées en personnes spécifiques, reconnues en personnages, du show télévisé. Agamben précise que, dans la pensée médiévale, reconnaître une image et se reconnaître dans une image, était appelé « amour » voire "désir ». Ainsi l'Homme sans nom, sans doute parce qu'il est l'innommable, est-il une entité spéciale méconnaissable, une personnification du mal d'amour, pour reprendre une expression chère à Pacôme Thiellement. L'Homme sans nom est la spécialité comme intentionnalité obsessionnelle: tendance à s'approprier exclusivement la Lumière du cœur de l'île pour fuir ce lieu et le détruire par la même occasion, pour le dérober au regard. Si l'île et la Lumière qu'elle recèle sont entendues comme des synecdoques de l'univers du récit et de l'éclairage qu'il peut donner de notre monde commun, sa visibilité, alors le monde de l'Homme sans Nom, est celui de la fiction comme pur divertissement, d'une diversion hors du commun. C'est un simulacre voué à être détruit pour passer à autre chose, s'échapper, en finir (leave plutôt que move on), la valeur d'exposition d'une société du spectacle :

14 Le spectacle est la séparation de l'être commun, l'impossibilité de l'amour et le triomphe de la jalousie ${ }^{11}$. 

le remède se trouvait déjà dans ce survival à rallonge qu'était Lost. À l'initial, Lost a été écrit à partir de l'idée que l'île serait un personnage à part entière et que son cœur recélerait une trappe. Cette idée fut proposée par J.J. Abrams avant de s'occuper de Mission Impossible III (2006) et de laisser Cuse et Lindelof se débrouiller avec le reste de l'intrigue dès la seconde saison. Charge à ces derniers de desceller la trappe de la série et de courir le risque d'assumer l'ouverture de ce mystère initial. Or dès les premiers épisodes de la saison 2, la série est quasiment pliée. En effet, nous est présenté le dispositif du Cygne et de son bouton sur lequel appuyer toutes les 108 minutes pour sauver le monde, puis, peu après dans le cours de cette même saison, est découverte la station de la Perle censée observer l'implication des candidats dans l'accomplissement de ladite tâche qu'ils croient être d'une extrême importance. Ce faisant, par le truchement des deux stations, les showrunners nous annoncent la fin du récit mais aussi et surtout l'objectif de Lost en tant qu'expérience télévisuelle. En effet, il s'avère que le test porte tout autant sur celui qui regarde depuis la Perle que sur celui qui est regardé dans le Cygne. Ainsi, regarder une fiction n'est pas une opération supposant une relation totalement asymétrique avec celui qui la montre. Regarder une fiction, c'est consentir à être interpelé par elle en étant prêt à donner du temps, pas simplement à le prêter. La narration télévisuelle engageant la responsabilité du spectateur l'incite ainsi à un certain régime réceptif pour entretenir une bonne distance avec l'émetteur, avec les impersonations qu'il a sous les yeux et qu'il accueille en donnant du temps. Une étrange réciprocité conditionne ce don qu'est la fiction télévisuelle. D'où ce que nous appelions l'oscillation du Cygne et de la Perle ${ }^{12}$. Cependant, exhiber cette oscillation n'est pas sans conséquences. Si le sens de la fiction télévisuelle dépend de la participation intime du téléspectateur, sans elle, le récit, comme le roi, est nu. Lost révèle corrélativement que la fiction télévisuelle peut relever d'une logique de conditionnement ou d'expérimentation psychosociologique de type Milgram où s'enchaînent, avec plus ou moins de bonheur, des clichés sans rime ni raison. Ceux-ci sont interprétés comme formant un ensemble cohérent, une intrigue où les péripéties répondent à une téléologie, à force de répétition et par soumission à ce qui sera fantasmé comme une autorité. Cette autorité omnipotente n'est rien d'autre que le revers d'un désir frustré d'être spécial dans un sens strictement spécifique, c'est-à-dire propriétaire exclusif d'une qualité me rendant absolument unique, occultant la dimension spéculaire du terme spécial présupposant une relation à l'autre. Cet autoritarisme est le revers d'un ressentiment, et l'illusoire perspective d'une échappatoire à notre dégoût de la désespérante vie commune...

C'est pour cette raison qu'est précisé, dès cet autre commencement qu'est sa saison 2, que Lost en rendra malade certains, d'où l'avertissement de Quarantaine écrit sur la trappe du Cygne. Il y aura une fièvre Lost, un emballement amenant à une montée aux extrêmes ${ }^{13}$. Or cette maladie d'amour ou Lost fever est, bien sûr, l'anagramme même de la série suivante, The Leftovers. Celle-ci est donc un Lost malade, celui de la quarantaine de la trappe de la Station du Cygne, où nombre de motifs sont repris et inversés pour répondre à et de la lecture hostile ${ }^{14}$ et ses critiques négatives, à l'ingratitude de sa réception. Les professions des personnages principaux de The Leftovers, Laurie, la psychologue, Kevin, le policier, et Matt, le prêtre, sont celles des personnages de la queue de l'appareil, Libby (Cynthia Watros), Anna Lucia (Michelle Rodriguez) et Eko (Adewale Akinnuoye-Agbaje) qui, dans la saison 2, se font un trip Lord of the Flies ${ }^{15}$ pour reprendre les mots de Charlie (Dominic Monaghan), et échouent à former une 
communauté en raison de leur violence. Le personnage le plus constant de The Leftovers, le fils Murphy, Michael (Jovan Adepo), porte le même prénom que le traître, Michael Dawson (Harrold Perrineau), de la saison 2 de Lost.

The Leftovers est néanmoins aussi un Lost brûlant car cette inversion des antagonismes, explicite l'opposition entre sens et non-sens de la première série, et en font donc une parodie extrême de celle-ci. Elle incite ainsi les gardiens du canon lostien, les candidats à la garde de l'île qui trinquent à la bouteille jacobite, à se réinterroger sur ce qu'ils gardent et donc sur leur propre exigence de récit. Qu'ont-ils regardé de Lost ceux qui ont des oreilles pour entendre, c'est-à-dire qui s'attèlent à interpréter la série? Se souviennent-ils que les derniers mots de la série étaient tenus par Christian (John Terry) et Locke, les deux incarnations principales du double de Jacob du Monstre de fumée « gargantuesque » comme dit encore Charlie dès le Pilote (S01E01) ?

Dans Lost, les showrunners nous offraient des chiasmes de noms d'images et d'images de noms. Ils travaillaient ainsi le paradoxe de la parole pure, le nom de feu (pur) magique et intime : celui qui appelle à être, celui qui nomme un être spécial comme spécial (en son unicité, sa transcendance), mais dans un vocable qui soit universellement communicable par-delà les divisions des langues et le fantasme d'un langage total l'appel à la grandeur commune. Ainsi usaient-ils du procédé de l'homonymie, d'abord avec des homonymes de grands hommes, puis, lors de la saison 2, à l'occasion de l'opération transmédiatique Lost Experience, ils étendaient cette contrainte d'écriture aux patronymes même des acteurs comme celui de Michael Emerson y interprétant le faux Henry Gale dénommé Benjamin Linus à partir de la saison 3. Emerson est homonyme du philosophe américain Ralph Waldo Emerson, le père caché voire refoulé dans la pensée américaine selon Stanley Cavell. Ainsi le récit présentait-il une réflexion sur l'impersonation et la responsabilité télévisuelle faisant du show une expérience de l'économie des médias audiovisuels ${ }^{16}$.

Identiquement, The Leftovers s'interroge sur celles-ci avec le special guest qu'est le personnage de Mark Linn-Baker jouant son propre rôle. Ce dernier regrette la disparition de l'équipe de la série Perfect Strangers diffusée sur ABC de 1986 à 1993, la chaîne diffusant Lost, sa persona de Larry Appleton apparaissant à l'écran et son identité civile étant entrelacées. L'épisode "Don't Be Ridiculous » (S03E02) a d'ailleurs comme générique celui de la série Perfect Strangers, le titre lui-même étant l'une des dernières répliques de Balki (Bronson Pinchot) dans l'épisode «Up, up and away " (S08E06) de la sitcom. Le jeu d'incident de frontière avec la rampe ou le quatrième mur, interrogeant la ressemblance spéciale des univers poétiques et commun, leur contamination réciproque, nous fait entrer dans un rêve audiovisuel partagé tant dans Lost que dans The Leftovers. Mais dans cette dernière série la matière dont est fait ce rêve relève moins d'un mourir ensemble lévinassien que du suicide collectif. En effet, Baker/Appleton contacte Nora pour la recruter, mais aussi pour qu'elle soit témoin qu'il va passer de l'autre côté c'est-à-dire très probablement se faire vaporiser. En lui disant qu'il veut rejoindre son équipe, sa famille, outre le fait qu'il cite dans l'esprit les dernières répliques de Larry dans "Up, up and away", et qu'il explique comment, scientifiquement, il compte faire, il se pose en témoin et médiateur pour que Nora passe le mot à son tour avant de se faire filmer (shoot) face caméra. Comme si, pour être accepté par les Dr Eden et Bekker, il ne fallait pas simplement consentir à l'éventualité de mourir mais parrainer un tiers, se tuer pour le subjuguer. C'est d'ailleurs ce que fera Nora en s'adressant à la caméra et donc en hors-champ aux deux physiciennes, mais 
aussi aux spectateurs dans la première séquence du «Book of Nora». Cependant, en prononçant les prénoms d'Erin et Jeremy, ses autres à elle, elle nous appelle à aller de l'autre côté et à nous efforcer de voir le show de la série autrement.

Une autre manière de reproduire la Lost Experience et ses jeux de noms et prénoms, c'est pour The Leftovers de représenter l'acte du baptême. Celui-ci un des paradigmes austiniens $s^{17}$ de l'usage du performatif, et sa représentation ré-ouvre la réflexion sur la dénomination et l'impersonation audiovisuelle proposée par Lost. Il était offert aux personnages de Lost d'être spécialement nommés car candidats à la reprise de la responsabilité de l'île, en buvant l'eau de la source de la Lumière et en recevant le « Now you're like me. » de son prédécesseur instituant une bonne réciprocité entre personnage et démiurge, et par voie de réflexion entre téléspectateur et showrunner.

Or cet acte est des plus traumatisant dans The Leftovers. Lorsque Kevin Garvey se fait baptiser et est donc censé recevoir son nom pur celui qui le rend intimement responsable, lors de l'épisode "The Book of Kevin » (S03E01), il a un flash de l'hôtellerie, de l'autre monde. Or ce flash met en crise la réalité même de ce que nous tenions pour le quotidien de nos héros à Jarden puisqu'on est en droit de se demander si tout ce que nous observions n'est pas une rémanence, un simulacre, les souvenirs pré ou postmortem de Kevin qui n'aurait jamais quitté l'hôtel et s'aperçoit qu'il est mort, n'est jamais revenu à la vie et n'a pas renoncé à être un assassin. Rappelons que Justin Theroux enrageant sur la part audiovisuelle de son identité, les souvenirs fragmentaires d'un moi qui se révèle par l'impuissance de son désir et son désir d'impuissance dans une chambre d'hôtel, est le thème du clip d'Hysteria (Matt Kirby, 2003) de Muse. Hysteria est probablement la matrice du personnage orphique de Kevin si l'on en croit la pochette de l'album Absolution. Muse est le nom du disciple d'Orphée dans la mythologie grecque et l'absolution est justement ce à quoi l'homme n'a plus accès avec ce parasite du christianisme qu'est la sécularisation de la religion et du sacrifice selon l'analyse d'Agamben du fragment posthume de Benjamin, «Le capitalisme comme religion». Le penseur italien distingue ainsi d'une part la profanation qui neutralise ce qui est sacré et sa logique de contagion, pour l'offrir à l'usage commun, qui sacrifie le sacrifice et donc purifie, de la sécularisation d'autre part. Celle-ci refoule le sacré et le reconduit dans un autre lieu, pour le rendre Improfanable, Innommable ${ }^{18}$. La profanation s'efforce donc de déparasiter le mystère religieux par la parodie.

22 Cette hôtellerie a tout de l'autre lieu, elle apparaît dans l'épisode "International Assassin " (S02E08) où Kevin n'a pas son nom pur, mais un pseudonyme digne de William J. Hyperbone, le personnage orphique du roman de Jules Vernes, Le Testament d'un excentrique (1899). Du G initial de son patronyme, il suffit de passer à la lettre suivante dans l'alphabet, $\mathrm{H}$, pour avoir son nouveau nom, Kevin Harvey. Or dans l'épisode "Crazy Whitefella Thinking " (S03E03), nous apprenons que le père de Kevin (Glenn Scott) s'appelle lui-même Kevin, et qu'en transportant le livre de son Fils, il amènera une femme, Grace (Lindsay Duncan), à mettre à l'épreuve sa foi en ces Écritures et abattre un shérif australien appelé lui-même Kevin... interprété par un acteur australien dénommé Damien Harvey. Le mauvais Kevin assassiné par Grace est donc le vrai Harvey qui est l'autre Kevin, l'assassin international de l'hôtel des visions post-mortem dans le récit.

Cet entrelacs des références réelles et fictives, d'images de noms et de noms d'images, donne au show une dimension agonique et apocalyptique comme si l'intrigue était, à 
l'instar de ce que put se révéler être la fin de Lost, la projection d'une expérience de mort imminente commune. Mais, comme nous venons de le voir, là où Lost prétendait nous exposer à un mourir ensemble, The Leftovers s'axerait plutôt sur un personnage refusant le let go, s'échinant à accuser son entourage, voire à assassiner, pour continuer à le hanter. Le récit ainsi compris est donc, à l'inverse de Lost, l'exhibition de la part suicidaire et homicide du monde, de son inclination à la violence, à tuer l'autre pour faire communauté, faisant de la fiction télévisuelle une mythologie à fonction sacrificielle pour des regards assassins, irresponsables et hypocrites. En d'autres termes, The Leftovers rappelle que la télévision est une lentille déformante en dénonçant un regard persécuteur sur le monde.

\section{Lens} émissaire déjà préfiguré dans la saison 2 par l'épisode Lens centré également, pour part, sur le personnage de Nora. Nora Durst, surnommée Nora Curst (Maudite) par des journalistes locaux dans l'épisode « Don't be ridiculous » (S03E02) et interprétée par la bien prénommée Carrie Coon $^{19}$, est le personnage qui, quantitativement, a le plus perdu lors du Sudden Departure. Ses deux enfants, Erin (Ella Taylor) et Jeremy (Anthony Cieslak), et son mari, Doug (Sebastian Arcelus) se sont volatilisés le 14 Octobre. Ces derniers s'évaporent alors qu'elle attend un appel pour un emploi qui la sortirait de son rôle de desperate housewife et qu'elle vocifère après sa turbulente progéniture dont le père prend le parti. L'absence de ses proches lui est donc d'autant plus insupportable qu'à ce moment ses désirs deviennent réalité. La contiguïté de son état subjectif, sa colère et son désir de ne plus les voir, avec le fait de leur disparition, l'amènent à penser que ledit désir est la cause de l'incident. Elle se cloue elle-même au pilori d'un vieux rouleau de sopalin qu'elle refuse de changer, relique de son ire contre sa fille qui renversa du jus d'orange sur son téléphone portable au moment où il vibrait. Ainsi refuse-t-elle aussi d'être réduite à l'identité de victime du Sudden Departure et surtout ainsi refuse-t-elle l'interprétation cathartique que la communauté de Mapleton produit de son statut de victime ${ }^{20}$.

Elle s'accuse de la disparition, d'où ses tendances dépressives et suicidaires, l'un de ses jeux sadomasos favoris étant d'engager des prostitués pour, revêtue d'un gilet pareballes, se faire tirer dessus. Or, son métier, enquêtrice pour des assurances couvrant les proches des Disparus (departed), l'amène à se méfier de ceux qui se déclarent victimes de l'incident. Inclassable au sein de la communauté, pas à sa place dans son rôle attendu, elle est, selon le titre d'un épisode lui étant consacré, Guest (S01E06). Elle est invitée mais spéciale. Comme si là où l'anglais marquait une distinction entre guest et host, elle assumait l'ambivalence latine du mot « hôte ", à la fois accueilli et accueillant, mais aussi « hostile ", autre - double-entendre se retrouvant dans Lost. Nora est tout et son contraire à la fois. Elle est, d'une part, objet de la commisération de ceux qui ont moins ou pas perdu de proches lors du 14 Octobre, aimée de ceux qui ne sont pas susceptibles de comprendre sa perte. Mais d'autre part, elle est aussi l'objet du mépris de ses semblables, de ceux qui seraient susceptibles de comprendre sa perte. Néanmoins, elle s'efforce bon an mal an de survivre, voire de reconstruire une cellule familiale à Jarden dans la saison 2, grâce à l'argent de la vente de sa maison, point chaud du Sudden Departure. 
26 Nora est l'un des personnages principaux du livre de Tom Perotta dont le récit audiovisuel est l'adaptation. Le roman s'achève à peu près là où finit la première saison, donc assez bien pour elle. La fin de l'épisode « The Prodigal Son Returns » (S01E10) est d'ailleurs plutôt comparable à celle du livre pour ce qui est du sort de ce protagoniste : elle trouve en Lily, la fille de Christine (Annie Q) et du thaumaturge, ou escroc, Saint Wayne (Joseph Paterson), la pierre d'angle d'un nouveau foyer aux côtés de Kevin Garvey (Justin Theroux) le chef de la police de la petite ville de Mapleton ${ }^{21}$. Toutefois les deux saisons suivantes, dans lesquelles, nous semble-t-il, sourd l'ascendant de Damon Lindelof (et de Nick Cuse, le fils de Carlton, l'autre showrunner de Lost) sur l'écriture, s'acharneront à ruiner ce projet. Celles-ci accusent, par effet de répétition, l'ambivalence de sentiments que provoquent son malheur et sa situation professionnelle. Le malaise qu'elle suscite monte clairement d'un cran dans la deuxième saison.

En effet, elle accepte de vendre sa maison de Mapleton, car, grâce à un chercheur du MIT, elle met l'incident sur le compte de causes géophysiques objectives, manifestement indépendantes de sa volonté et dont elle ne saurait être raisonnablement responsable: c'est la maison qui était un point chaud concentrant anormalement certaines ondes émises lors du Sudden Departure. Or cette explication, parce qu'elle suggère que certains individus sont des "lentilles ", se retourne contre Nora dans l'épisode "Lens ». À ce titre, ils concentrent le rayonnement d'ondes ayant fait disparaître les departed. À l'énoncé de ces faits qui attesteraient qu'objectivement Nora est la cause de la disparition de ses proches, s'adjoint, brutalement, une récrimination donnant auxdites ondes et à leur focale une valeur morale et sacrée : Nora est responsable mais encore coupable de la perte des siens car elle est l'incarnation d'un ange déchu, d'un messager dévoyé, Azraël. Le choix de l'intitulé de l'épisode n'est pas anodin: toute optique de caméra suppose au moins une lentille concentrant dans l'appareil ce phénomène ondulatoire-corpusculaire qu'est la lumière traduite en signaux électriques à l'écran. Par voie de réflexion, The Leftovers renvoie à ses conditions réelles de production et de prise de vue, mais aussi d'écriture. L'intrigue désignant ici la focalisation à partir de laquelle se voit le récit, un mauvais médium qui déforme l'intrigue afin de lui faire dire autre chose que ce qu'elle dit, de cacher son propos. Toutefois ce mauvais médium est-il vraiment Nora? Ne serait-ce pas notre propre tendance de spectateur à relire les malheurs de Nora en les attribuant à une persona de substitut cathartique, c'est-à-dire à la construire non pas comme victime du Sudden Departure mais comme irascible poissarde donc bouc émissaire coupable de ce qui lui arrive? La soudaine irruption de cette sentence a tout d'un délire, soit de la part de celui qui le profère, soit de la part de Nora hallucinant mélancoliquement des invectives confirmant le sentiment d'indignité qui la taraude. Le reste de la fiction corrobore l'hypothèse du délire invraisemblable de l'interlocuteur puisque celui-ci prétend être au service du Vatican mais que nous ne verrons aucun membre de l'Église catholique perpétuer l'accusation ensuite. Néanmoins, un protagoniste tenant un rôle majeur dans la narration, est un pasteur intimement lié à Nora. Or l'une des modifications significatives de l'adaptation de Lindelof du roman de Perotta, est d'avoir fait du pasteur Matthew Jamison non pas l'ami, mais le frère de Nora. Tous les deux sont orphelins de père et de mère depuis l'enfance. Matt se substitue ainsi au personnage romanesque de la sœur de Nora, Karen, banlieusarde de classe moyenne tentant de lui redonner goût à la vie normale, soit à la vie domestique de banlieusarde de classe moyenne. 

envoie l'un des deux boucs, tiré au sort, qui a reçu tous les péchés du peuple, le jour du Grand Pardon (Yom Kippour). Cet exil s'inscrit dans un rituel décrit au chapitre XVI du livre du Lévitique. Le substitut sacrificiel exposé à l'assemblée par un prêtre transférant sur ses cornes les signes des iniquités communes, est voué à mourir dans le désert. Ce rite est l'une des origines possibles de l'expression commune «bouc émissaire », qui désigne de façon profane la victime, souvent innocente, d'une persécution collective arbitraire ${ }^{22}$. Dans le roman de Perotta, Nora, au détour d'une promenade forestière à vélo, aperçoit entre autres apparitions étranges une horde de chiens, un homme poussant un fauteuil roulant vide, un pseudo prêtre catholique illuminé à la barbe poivre et sel, et enfin un homme égorger un mouton ${ }^{23}$. Cette dernière vision se retrouvera dans l'adaptation télévisuelle dans la saison 2, cependant, elle est modifiée. En effet, dans la série, nous retrouvons un habitant de Jarden, la ville où personne n'a disparu le 14 Octobre, Jerry (Cody Haynes), qui égorge, non pas un mouton, mais une chèvre.

Dans ce même épisode, "Lens », après l'accusation du Vatican portée contre Nora d'être Azraël, Jerry s'apprête à faire son œuvre. L'explication mythologique du phénomène social s'accompagne donc du rituel sacrificiel: le substitut, objet d'un sacrifice violent chasse le mauvais œil guettant la communauté, afin d'en régénérer l'ordre et affermir son identité. Le substitut se voit par là-même transcendé, exhaussé de son statut de victime à celui de divinité, de démon. Ce fait religieux est démonté dans ces mécanismes psychosociaux par Erika (Regina King). Celle-ci refuse qu'en pleine assemblée, Jerry tue régulièrement (selon des règles et rituellement) une chèvre pour la seule raison qu'il le fit le 14 Octobre, au moment de la catastrophe. Erika, le médecin de la ville, prend à parti la communauté en dénonçant l'obscurantisme religieux hypocrite et mercantile de Jarden.

Sa diatribe expose l'économie des rituels et de la mystification par laquelle les habitants se croient rescapés pour vendre leur ville comme Miracle aux profanes, aux autres. Dans la lignée de l'explication positive que livre Frazer des rituels de sacrifice de substitution parmi lesquels celui des Saturnales romaines dans Le Bouc émissaire en $1913^{24}$, Erika entend démontrer que la pensée magique n'est qu'un raisonnement inexact dont est victime la communauté. Les habitants de Jarden sont les jouets d'une illusion téléologique: ils intervertissent le rapport de cause à effet. Des faits objectivement contigus à la catastrophe (tuer une chèvre, se marier) associés à des sentiments de joie subjective en accord avec les désirs des habitants d'échapper à la disparition du 14 Octobre, ont été instaurés de façon imaginaire, fort improbable, au rang de causes de l'évènement. Ce qu'ils faisaient alors qu'est arrivée la catastrophe dont ils s'estiment épargnés, est devenu pour eux, à force de répétition et d'autoconditionnement, la cause de leur salut. Par voie de réflexion, cette intervention d'Erika vaut également, comme interpellation des showrunners à l'assemblée que nous sommes, nous spectateurs. Ne sommes-nous pas conditionnés, par l'agencement des péripéties que nous regardons, à croire en un sens total et achevé de l'intrigue qui ne se trouve que dans notre incapacité à éteindre notre télévision et notre désir d'être consolé de notre impuissance à changer notre quotidien en nous focalisant sur des substituts sacrificiels, voire des victimes expiatoires ? Cet opium religieux idéologique auxquels la fiction et le médium télévisuel s'apparentent était déjà exhibé comme tel dans Lost par ses statues de la vierge bourrées d'héroïne contenues dans le biplan cachant la station

TV/Series, Hors séries 1 | 2016 
de la Perle. Cependant, comme dit précédemment, en exposant ainsi ce processus d'aliénation, Lost nous invite à voir la fiction et la télévision autrement et l'on peut en déduire que The Leftovers reproduit le même canevas sinon dans l'épisode « Lens », du moins dans l'épisode « The Book of Nora ».

En effet dans l'épisode «Lens », le discours d'Erika n'est pas exclusivement rationnel. En tant que mère, son propos est motivé par la disparition de sa fille Evangeline (Jasmin Savoy Brown). De surcroît, son comportement s'inscrit lui-même dans une logique sacrificielle, puisque la première séquence installant son personnage dans l'épisode " Axis Mundi » (S02E01) nous la révèle enterrant des moineaux trois jours durant, pour voir s'ils survivront et donc si ses vœux se réaliseront. En effet, les deux événements sur lesquels se cristallisent la communauté pour se représenter l'irreprésentable de la catastrophe ne sont pas anodins : un meurtre et des noces. L'élimination de la Bête pour l'avènement de la Belle, est la synecdoque de la représentation sacrificielle de la catastrophe : il a fallu que beaucoup disparaissent pour que Jarden soit épargné, c'est-àdire pour que Jarden soit Autre, pour que les membres de l'assemblée puissent faire alliance entre eux et que l'assemblée dans son ensemble fasse alliance avec la violence de la catastrophe et se fasse un Nom, Miracle.

Le sacrifice violent de meurtre ou d'expulsion du démon donnant lieu à l'avènement de noces et de l'épouse est une représentation de l'ambivalence du désir, ce qui nous semble s'inscrire dans la généalogie de la culture telle que la décrit Girard dans La Violence et le Sacré (1971) ${ }^{25}$. Selon ce dernier, le rite est la répétition d'une crise sociale originelle qui a mené au massacre d'une victime tout à la fois honnis et adorée par sa communauté la considérant à la fois comme Belle et Bête. Or on ne peut connaître cette victime en tant que telle qu'en déconstruisant les mythes qui la posent comme responsable de la crise car racontés par des bourreaux s'exonérant de la responsabilité de leur meurtre et en le justifiant, mais aussi en déconstruisant la logique de la représentation qui recèle en elle une forme de substitution. Dans The Leftovers cette crise en son irreprésentabilité nous paraît être le Sudden Departure qui demeure un mystère, une absence qui travaille et hante toute la communauté comme sa responsabilité et dont Nora va servir de substitut expiatoire sur laquelle tout le monde se focalise pour que sa souffrance donne sens et pérennise l'irresponsabilité commune.

Cette ambivalence de la représentation du désirable comme Belle et Bête est soulignée dans une séquence, apparemment plus apaisée, de l'épisode "The Book of Nora » lors du mariage d'Agie (Alison Bell) et Eddy (Damian Hill). La cérémonie de Jarden préfigure la cérémonie australienne car l'opposition de la Belle et la Bête y est répétée, explicitée, et sa polarité accentuée. Elle est répétée car la chèvre est exhibée dans l'assemblée au moment où l'époux s'adresse à la mariée en prenant l'assemblée à témoins. Elle est explicitée car le rituel est moins sanglant et semble moins arbitraire, comme le remarque Kevin : "At least, they didn't sacrifice it.». En effet, le rituel passant par la parole assigne un sens symbolique à l'expulsion de l'animal ; celle-ci sert alors de base à un engagement verbal de l'époux à sa femme et donc à un contrat de mariage avec obligation réciproque. L'opposition, enfin, est accentuée : la logique d'expulsion de la chèvre, reconnue, transcende l'alliance et la promesse du mariage. La reconnaissance de l'expulsion s'accompagne d'un échange de vœux différé par un lâcher de colombes baguées de messages secrets à destination d'inconnus, d'une part, et par la perspective de leur retour, d'autre part. 
Or cette cérémonie fait office de pseudo-premier rendez-vous pour Kevin et Nora, selon celui-ci. En effet, ce dernier suggère à l'élue de son cœur d'entrer dans son jeu et de faire comme s'ils se rencontraient pour la première fois. Pourtant, dans les faits, c'està-dire dans ce qui a été raconté dans les deux premières saisons de la série, donc d'après leur mémoire et celle du spectateur, ils ont été ensembles quatre ans durant et se sont violemment séparés, il y a de nombreuses années, dans l'épisode "G'Day Melbourne » (S03E04). Le temps écoulé avant ces retrouvailles fait d'ailleurs l'objet d'une ellipse au cœur de l'épisode. symboliser leurs péchés. Dans un discours édifiant, un argument moral rationnel, l'époux, Eddy, oppose les péchés dont le sujet agent est responsable (choisir le mal pour le mal en vertu même de son libre arbitre) et la faute dont le sujet agent n'est que relativement responsable (choisir le mal en le prenant pour le bien par défaut de jugement voire de capacité physique). Il s'engage, alors, à fauter avec son épouse, mais à ne plus pécher avec elle. Cependant, quelques secondes après, le même Eddie fera fi de ladite distinction conceptuelle, supposant une certaine définition du libre-arbitre. Si le rituel $\mathrm{du}$ bouc émissaire est celui auquel se livrent showrunners, acteurs et spectateurs en regardant la télévision, le libre-arbitre conceptualisé par Eddy renvoie au rapport du spectateur du récit télévisé à sa suspension volontaire d'incrédulité. Dans quelle mesure choisissons-nous de croire ou non à une fiction télévisée ? Ce concept n'est-il pas une manière rétrospective de justifier le rituel cathartique de la fiction pour continuer à la réaliser ? Se faire croire qu'on a le choix, le droit de regarder ou non sa télévision n'est-ce pas la meilleure manière de continuer à la regarder sans interroger l'économie de ce médium?

Or, comme la chèvre avant la cérémonie, Nora n'a pas de collier. Comme la Bête à expulser pour que la Belle puisse se marier, cette trouble-fête fait valoir une autre version de cette promesse de fidélité conjugale. Une version dans laquelle la souffrance est éprouvée par l'expulsée afin que la communauté vive. En effet, elle refuse la logique attribuée à Caïphe dans le chapitre 11 de l'Évangile de Jean selon laquelle il est nécessaire qu'un homme meure pour le bien de la communauté, que l'on retrouvait mutatis mutandis dans Star Trek into Darkness (réal. J.J. Abrams, 2012) dans le conflit entre Spock (Zachary Quinto) et Kirk (Chris Pine) et à la fin de l'épisode « Live together, Die alone » lorsque Desmond se sacrifie pour Penelope ${ }^{26}$. La souffrance de l'expulsée est toujours injuste. La communauté ne peut s'exonérer pas de sa responsabilité par une justification strictement quantitative selon laquelle la vie de plusieurs vaut juste plus que celle d'un seul. Elle ne devient vraie communauté (et non simulacre de société, agrégat d'individualités ou totalitarisme tyrannique) qu'en regardant en face ce qu'elle méconnaît, c'est-à-dire la monstrueuse disqualification de l'autre à laquelle elle a procédé. Aussi Nora refuse-t-elle comme mensonger le récit de Kevin faisant table rase de leur passé commun. Elle refuse la manière dont il raconte cette soirée comme étant leur premier rendez-vous et le commencement d'une histoire censée être finie - au même titre donc que la série dont c'est le dernier épisode. Cela étant, ce refus tient tout autant d'une lucidité que du fait que Nora tient elle-même à un mensonge sur lequel elle prétend avoir refait sa vie. Depuis sa séparation d'avec Kevin, elle se fait appeler Sarah et est devenue receleuse de colombes au service d'une bonne sœur (Linda Cropper) qui les loue pour les invités de noces; ces derniers y accrochent leurs vœux

TV/Series, Hors séries 1 | 2016 
avant de les laisser s'envoler, en attendant une réponse d'un inconnu, d'un autre. Néanmoins elle ne se fait pas d'illusion sur l'arnaque qu'elle monte avec la religieuse.

Néanmoins, lors du climax de ce dernier épisode, Nora, au bout de la nuit, retournant chez elle en vélo, chute et aperçoit la chèvre coincée, les colliers pris dans les fils de fer de la clôture d'un champ. Nora remonte la pente, après avoir dégringolé, et libère l'animal sur le thème de November de Max Richter. Hors-champ, tonne la foudre. Elle prend ses perles, donc les péchés de la communauté pour qu'alliance donc société il y ait. En se substituant au substitut auquel sont attribués les maux des autres, elle reconnaît l'accusation que porte son propre regard puisqu'elle mettra les perles sur son rouleau de sopalin en mémoire de ses enfants. La séquence est une révélation psychologique individuelle comprise dans la diégèse: Nora s'aperçoit alors qu'elle refusait de se pardonner le Sudden Departure car, intimement, elle en accusait aussi ses proches, Erin et Jeremy, dont les prénoms étaient si difficiles à prononcer au début de l'épisode, et dont Lily ne fut qu'un substitut de cet inavouable reproche. Enfin, elle est prête à aller de l'avant (move on), à prendre le risque d'aimer Kevin et se voit alors en mesure de faire le récit de sa propre disparition et surtout d'élucider le mystère du Sudden Departure, en requérant de la bienveillance de son bien aimé qu'il y prête foi et qu'ils puissent enfin se marier : tout est bien qui finit bien. Ainsi, en libérant la chèvre, il nous semble que Nora n'assume pas seulement sa part d'ange déchu par une contrition spectaculaire, une confession publique qui ne fait reconduire celle-ci d'une autre manière, mais qu'elle profane, c'est-à-dire qu'elle expulse la logique d'expulsion et de substitution même. Seule avec l'isolée qu'est la chèvre, elle incite le regard du spectateur à se singulariser également, se faire intime en reconnaissant sa solitude, sa misère sans autrui, pour pouvoir faire société et donc voir autrement le Sudden Departure.

\section{See you in another life, brother}

Cette antépénultième séquence est-elle une imitatio christi? Avant de choir de son vélo, la musique en off ponctuant le retour de Nora est le morceau Me, myself and I interprété par Billie Hollyday. Elle pourrait être entendue comme une allusion quelque peu ironique à une psychologie trinitaire image d'une Trinité divine : Père, Fils et Esprit, ce dernier étant figuré par la Colombe. Cela dit, dans The Leftovers, ne serait-ce pas plutôt le personnage de Matt qui s'assigne de suivre un tel chemin de croix ? En effet, le frère pasteur de Nora, se présente de son propre chef comme substitut dans l'épisode « No Room at the Inn » (S02E05). Ainsi prend-il la place au pilori de celui qui y a été cloué. Quelle différence y a-t-il alors entre le frère et la sœur ? La substitution de celui-là est volontaire et glorieuse aux yeux de la cour des miracles des rejetés de Jarden. Matt met un point d'honneur à son humiliation et se rengorge d'un " antitriomphalisme triomphant ${ }^{27}$ " pour reprendre les mots de l'analyse qu'offre l'anthropologie girardienne du phénomène du bouc émissaire. Selon Girard, le rituel sacrificiel et le mythe qui l'accompagne supposent une double substitution: le substitut sacrificiel déterminé dans le cadre d'un rituel déjà structuré et défini qui est la répétition d'un meurtre collectif originel ; celui de la victime émissaire qui subit et calme arbitrairement la vindicte de la foule. Matt, de par son sacerdoce, est donc image du bouc émissaire et non bouc émissaire (ou victime émissaire). Il est substitut rituel du substitut expiatoire mais pas substitut expiatoire de la violence collective, donc, paradoxalement, son sacrifice 
officiel confirme et justifie la violence collective exercée officieusement contre des victimes expiatoires au premier rang desquels sa propre sœur.

rituels et mythes tribaux et en critiquant des tragédies grecques, la théorie suivante : le lynchage d'un individu, la rage de tous focalisé sur un, est à l'origine des rituels et interdits rendant possible une économie sociale viable. Selon cette même théorie, reprenant des hypothèses de la psychologie de la communication de l'École de Palo Alto, le caractère essentiellement mimétique du désir laissé à lui-même, mène à une recrudescence de doubles contraintes dans les relations humaines et à une schizophrénie galopante, une prolifération de doubles, une pandémie de violence, la guerre de tous contre tous illustrée par le thème mythique des frères ennemis ${ }^{29}$. À propos de la double contrainte et de Damon Lindelof, dans Lost déjà, Naomie (Marsha Thomason) apportait sur l'île le roman de Bruno Heller, Catch 22 (1962), dans l'épisode éponyme (S03E17). Or ce roman fut écrit pour part sur ce principe de communication et la série y interprétait manifestement l'expression Catch 22 (Entourloupe 22 en français) comme une exégèse de Genèse 22, le sacrifice d'Abraham, si l'on en croit le flashback de Desmond et son vin de La Moria ${ }^{30}$.

41 La guerre de tous contre tous, indissociablement économique et représentationnelle, est résolue par le meurtre collectif de celui qui est arbitrairement tenu responsable de ladite violence. Il est à la fois maléfique (suppôt de l'envie collective) et bénéfique (son cadavre établissant une concorde en différant et médiatisant la réciprocité mimétique), et est ainsi élevé au rang de dieu. Ce meurtre calmant la rage initiale est le socle d'un ordre social efférent. Il est au principe d'une conception de l'identité et de la responsabilité à l'image et à la ressemblance du cadavre de la victime expiatoire, le signifiant transcendantal. Repris et imité par l'institution du sacrifice et d'un second substitut, le substitut rituel, le lynchage initial fonde la culture d'une communauté : technologie, art, sciences, hiérarchie politique ${ }^{31}$.

Que par son attitude sacrificielle Matt confirme et prolonge le meurtre d'une victime qu'il se cache à lui-même et que celle-ci soit sa sœur, cela s'explicite lorsque November retentit en off, de la même manière que pour cette dernière. La musique rythme le dialogue du pasteur avec Dieu/David Burton, ligoté sur un fauteuil roulant, dans l'épisode sérieusement parodique "It's a Matt Matt world». Dans la soute d'un bateau accueillant une orgie nocturne, et en compagnie du « dieu » en cage de ladite orgie, le vieux lion Frasier, le pasteur demande en vain des comptes à Dieu/David Burton. En vain, car son interlocuteur fait reposer toute responsabilité sur la seule capacité plutôt que sur une quelconque justification morale ou métaphysique. Il accuse cette exigence de justification de n'être qu'envie et vaine soif de reconnaissance, un ressentiment de la part de Matt. Ce dernier eût voulu que son Dieu le jugeât et c'est pourquoi il le juge à son tour. Or ce Dieu ne juge pas, non parce qu'il aime au-delà de toute mesure, mais au contraire parce qu'il prétend être indifférent et amoral, donc injuste et irresponsable. Il 
n'assume, comme les showrunners pourraient le faire, que la responsabilité du Sudden Departure, c'est-à-dire l'élément déclencheur du récit de The Leftovers. Entendant cela, Matthew a une révélation apocalyptique: un souvenir de sa mort, c'est-à-dire de sa maladie d'enfant et de la rémission qui l'a amené à croire, au point de penser que la vie lui était due en lui cachant l'éminente possibilité et donc la gratuité de sa mort. Se rappelle à lui, de la sorte, la problématique signification de donner la mort ${ }^{32}$, voire du " Mon Dieu, mon Dieu, pourquoi m'as-tu abandonné?" du Psaume 22 où le juste redoute le bâillement des gueules des lions rugissants. L'ecclésiastique pose une question ouverte sur une responsabilité infinie, enfin entrevue : «Is that why you're killing me?». Celle-ci l'appelle à défaire son Dieu de ses liens, pour qu'il lui prodigue une ultime leçon fort ironique sur le salut en un geste parodiant l'absolution.

Par voie de réflexion, cela peut s'entendre comme une leçon que nous donnent les showrunners, les démiurges de la série, sur la différence entre la consolation critique et la dimension eschatologique du récit : comme Matt, les spectateurs attendant le sens tout d'un spectacle produit par un auteur omnipotent dans un monde de fiction ne font que projeter leurs propres frustrations et reconduisent une stricte séparation toujours plus frustrante entre récit et réel. Ce n'est qu'en reprenant le récit de lui-même, en le profanant pour se faire narrateur à son tour que le récepteur peut entendre ce qu'il peut espérer de la narration. Dès lors, Matt comprend-il qu'il n'avait agi jusqu'ici que par ressentiment, pour qu'à la fin des temps advienne l'image vengeresse, plus que justificatrice, qu'il se faisait de lui-même à travers l'Autre ? Saisit-il enfin qu'à vouloir faire l'ange, il s'était vénéré lui-même comme bête, roi des animaux, lion? Comme l'écrivait feu Michel Serres :

Le schéma girardien ne cesse, en effet, de se reproduire, comme systole et diastole; il se propage comme une onde: distributive, la violence maligne se localise; localisée, elle se redistribue pour se localiser à nouveau. Comme un lion rugissant cherchant qui dévorer, elle circule tanquam leo rugiens circuit quaerens quem devoret. ${ }^{33}$

Cela étant le lion est l'homonyme de Lyon, patronyme du sous-marinier français détruisant une île du Pacifique à coup de bombe atomique au début de l'épisode. Où se trouve cette île? En cartographie, les lions comme les dragons désignaient des régions inconnues et dangereuses. "Hic sunt dracones » n'était-il pas écrit sur la porte antiexplosion du Cygne dans Lost pour cartographier l'île ${ }^{34}$ ? Le rapprochement de cet épisode de The Leftovers avec l'épisode « The Incident » (S05E17) paraît alors fécond. En effet dans ce dernier épisode, comme au début de «It's a Matt, Matt world», les héros font exploser une bombe atomique sur l'île en 1977. D'autre part, en 2007, Benjamin Linus épaulé par le simulacre de John Locke qu'est le double de Jacob tue ce dernier, qui est le démiurge de l'île, après lui avoir reproché son irresponsabilité, c'est-à-dire d'avoir sacrifié sa vie pour lui sans espoir de rétribution. Identiquement Matt rencontre son Dieu/David Burton, et lui fera des reproches analogues, mais le Dieu de The Leftovers est très manifestement une cynique imposture doublée d'un assassin débonnaire. De surcroît Matt, contrairement à Ben, ne le tue pas car il entend ce qu'il lui dit. Ce n'est pas lui mais le lion qui dévore Dieu/David Burton. Or le lion est aussi l'un des quatre animaux du Temple de Jérusalem et, par extension, il représente Juda le fils de Jacob dans la Genèse, le Christ dans l'Apocalypse de Jean et aussi l'évangéliste Marc. Si l'on considère les prénoms et noms de l'interprète de Jacob dans Lost, Mark Pellegrino, le chant liturgique hébreux de l'épisode «It's a Matt, Matt world» qui précède la 
confrontation et la référence finale à Venise de la chanson d'Aznavour, Venise étant la ville de Marc suggèrerait que l'île n'est pas totalement détruite même dans The Leftovers. Cet épisode est-il l'annonce du retour de Jacob et d'un autre regard par la série sur le rôle de la fiction télévisuelle?

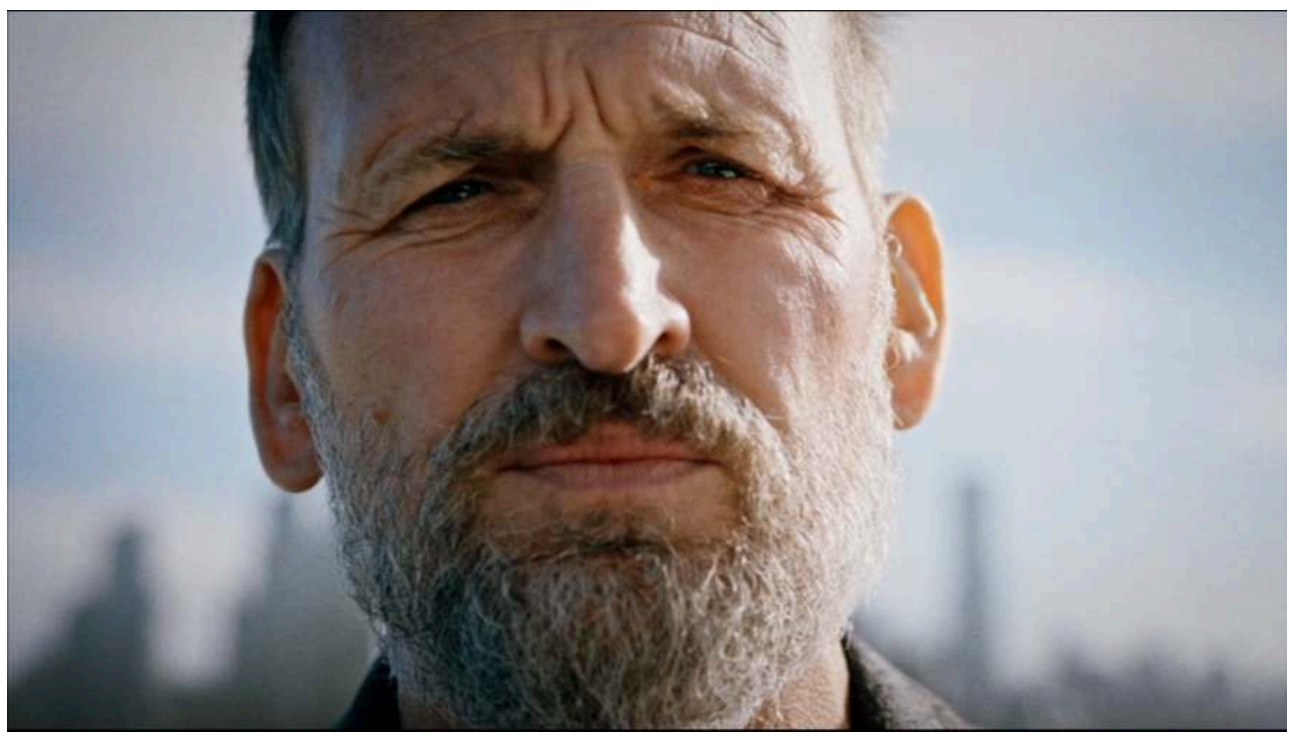

«It's a Matt, Matt world » (real. Nicole Kassel, 2017)

Toujours est-il que cette confrontation avec ce prétendu démiurge transcende Matthew comme aucun autre personnage de The Leftovers, sinon sa sœur au début de l'épisode 8 qui nous envisagera dès le premier plan. Matthew se convertit littéralement puisqu'il se retourne vers la caméra et donc vers le spectateur. En effet, l'entretien avec Dieu/David Burton rend Matthew apte à briser ce quatrième mur qui en faisait le héros d'un récit mensonger sur lui-même, et donc un mauvais messager, afin d'envisager une certaine réciprocité entre lui, celui qui filme et celui qui reçoit le film. Par ce renoncement, il interpelle le spectateur en hors-champ, se donnant à voir, assumant son visage, encrouté, mais souriant et libéré en disant : "That was the guy I was telling you about. » Il assume ainsi sa part de narrateur délégué de ce que fut jusqu'ici The Leftovers, révélant l'échange de Christopher Eccleston en Matthew Jamison et de Matthew Jamison en Christopher Eccleston dans la nudité de ce visage lui rendant sa dimension spéciale à la fois spécifique et spéculaire :

Spécial est en effet l'être (le visage, le geste, l'événement) qui, sans ressembler à aucun autre, ressemble à tous les autres. ${ }^{35}$

Par voie de réflexion, le spectateur envisage en cette interpellation le mensonge du « Book of Kevin », l'Évangile selon Kevin en français trompeur, puisqu'il s'agit plutôt de l'Évangile de Kevin selon Matt. Comme l'indique Sarah Hatchuel, le récit que fait Matt dans ce Book of Kevin n'est-il pas un indice métadiégétique grâce auquel nous pourrions relire le récit de The Leftovers ${ }^{36}$ ? En d'autres termes, l'Évangile de Kevin n'est pas uniquement le titre du premier épisode de la troisième saison, c'est aussi une certaine manière de voir l'intégralité de ce qui précédait dans la série, puisque la focale de Matt était sur Kevin au détriment de Nora. Damon Lindelof ne nous paraît pas dire autre chose lorsqu'il déclare :

We had the inklings of this kind of Life of Brian, reluctant Messiah construct. So we liked this idea of The Book of Kevin, and then the other thing that was 
up on the white board was just the word 'Nora. Nora. Nora.' I kind of felt like Season 2 really focused a lot on Kevin's journey, and I sort of felt like Nora Durst has the longest road to travel towards some level of healing and internal and personal salvation, and I want to go down that road because she lost so much $^{37}$. avant de lui faire chanter Homeward Bound de Simon et Garfunkel au karaoké, se révèlerait tout aussi bien n'être qu'une mystification. Dès lors par cette interpellation de Matt/Christopher Eccleston, «It's a Matt Matt world» est donc sérieusement parodique et révèle la double frénésie c'est-à-dire la dimension parodique extrême de The Leftovers par rapport à Lost. Comme l'écrit Agamben, la parodie est littéralement ce qui se tient à côté $d u$ chant, en contre-chant, c'est-à-dire n'est pas tout à fait dans la fiction, ni dans le réel, au seuil «entre les mots et les choses ». C'est ce que fait Matt/ Christopher Eccleston il est à côté du chant (du Cygne) qu'est Que c'est triste Venise, et apparaît dans la parabase de la série. Dans le théâtre grec, la parabase est le moment où les acteurs sortent de scène et que le chœur s'adresse directement au spectateur en s'avançant sur une partie de la scène appelée logeion, lieu du discours, $\log e^{38}$. L'interpellation de Matt/Christopher s'achève dans le dernier dialogue avec sa sœur dans l'épisode "The Book of Nora» où le révérend se focalise enfin sur celle qu'il a vraiment abandonnée pendant toute la série. «Qu'as-tu fait de ton frère? » était la question qui taraudait Jacob dans Lost, affirmions-nous dans un article précédent, "Qu'as-tu fait de ta sœur?" se dit enfin Matthew. L'abandon, le let go en termes lostiens, le dispose à une pure parole une improvisation, une nécrologie en cadavre exquis pour Nora où il devient Gecko pour amuser sa sœur en souvenir des lettres qu'il lui écrivait, petite, quand elle était au Camp du Saint Esprit... qu'elle appelait Camp Enfer et Damnation. Or cette improvisation agence dans son récit au moins deux termes, le nom d'une ville et un prénom. Ceux-ci laissent entendre, par voie de réflexion, que la nécrologie n'est pas simplement une plaisante galéjade révélatrice du capricieux inconscient de Nora. C'est une proposition de récriture et de relecture de certains événements de la série par les showrunners pour la mémoire des spectateurs.

Le nom de la ville proposée, Cairo, le Caire où, dans l'improvisation de Matt, Nora est censée être née et avoir fondée sa famille après ses études, est le lieu-dit de la cabane initiatique où Matt est allé chercher Kevin. Ce dernier y avait séquestré Patti Levin (Ann Dawd) avec Dean (Michael Gaston), tueur de chiens lunatique qui, dans le roman, vit à Sycomore Street. Matt aide Kevin à enterrer Patti après qu'elle s'est suicidée devant lui, événement que le pasteur n'a pas vu, mais auquel il a consenti de croire sur le seul témoignage de son ami. D'autre part, il est fait mention du prénom Antonio, dans la phrase " the great Antonio in the sky.", prénom qui, dans cette nécrologie fantaisiste, correspond au nom du dieu des Cieux, le Très Haut, que rejoindrait Nora après sa vaporisation par les Dr Eden (Katja Herbers) et Bekker (Victoria Haralabidou). Ce dieu paraît bien être, pour Matt, celui qu'il désignait auparavant comme " the guy that I was telling you about. », soit un supposé point de vue omniscient au récit de The Leftovers qui était en fait un point de vue interne perclus de ressentiment, la fausse conscience du pasteur diaboliquement hypostasiée. Cependant, en ayant vu Lost et sachant que cette série est très inspirée de The Tempest de Shakespeare, la référence à Antonio est éclairante. Antonio est le frère (jumeau ?) de Prospero, le maître de l'île dans la pièce de théâtre, auquel équivaut le jumeau de Jacob de Lost. Sa mention dans le cadavre exquis de Matt revient à faire de The Leftovers la parodie de Lost mais pas nécessairement son

TV/Series, Hors séries 1 | 2016 
simulacre. C'est un simulacre si l'on ne retient de la série que les événements rapportés par l'Évangile de Kevin écrit par le ressentiment partiel et partial de Matt. Toutefois, le fait de signaler et, ce dans une nécrologie, cette mystification, le fait d'en donner ainsi l'intelligence au spectateur, est aussi un recours à ladite mystification.

Or qu'y a-t-il hors l'Évangile de Kevin? Il y a l'Évangile de Nora. Dans Le Bouc émissaire encore, Girard distinguait entre les deux types de récits religieux : ceux à victimes ambivalentes et vengeresses que la violence collective divinise en cachant la persécution dont elles furent l'objet et ceux à victimes innocentes d'une persécution sans cause thématisée comme injuste - l'intelligence du cœur profanant cette persécution sans cause et l'Accusateur (Satan) dont elle relève, est la Sagesse, considérée comme inspirée, procession du Paraclet (la Colombe) :

Le bouc émissaire que le texte dégage pour nous est le bouc émissaire dans le texte et pour le texte. Le bouc émissaire qu'il nous faut dégager nous-mêmes est le bouc émissaire $d u$ texte. Il ne peut pas apparaître dans le texte dont il gouverne tous les thèmes; il n'est jamais mentionné en tant que tel. Il ne peut pas devenir thème dans le texte qu'il structure. Ce n'est pas un thème mais un mécanisme structurant ${ }^{39}$.

51 Dès lors, si les premiers récits sont racontés par les lyncheurs et s'ils justifient de la sorte l'acte commis, alors on ne saurait en comprendre le sens qu'à rebours de ce qu'ils professent littéralement voire même allégoriquement ${ }^{40}$. Ainsi présente-t-il la tradition brahmanique mais surtout judéo-chrétienne comme contrepied des mythes. Il analyse dès lors plus particulièrement l'Évangile de Marc comme révélation de la prétérition et $\mathrm{du}$ rebours qu'opèrent les mythes sur leur lynchage originel. C'est par ce double rebours profanatoire que se déconstruit la mystification et que se retrouve la vérité. Dans The Leftovers, le Livre de Nora nous parait jouer un rôle analogue par rapport au Livre de Kevin. Celui-là dont le titre achève vraiment la série, serait une version de l'intrigue racontée par un Matthew rebaptisé Gecko, soit à peine auteur, car mourant, avouant son angoisse et sa vulnérabilité, mais reconnaissant enfin en sa sœur ce qu'il avait oublié : qu'il la considère comme « the bravest girl on earth. » Que s'est-il passé dans l'ellipse séparant l'entrée de Nora dans la machine et ses retrouvailles avec Kevin? Ou plutôt, qui a passé ? Matt, le seul des personnages principaux à savoir si Nora est allée ou non de l'autre côté, ce tiers rendant possible les retrouvailles des amants.

Aussi ce lien de fraternité entre Nora et Matthew Jamison qui est très probablement le fait de l'adaptation par Lindelof du roman de Perotta, acquiert-il ici une autre dimension si l'on considère cette donnée en regard de Lost. En effet, dans cette série, nous retrouvons Desmond (Henry Ian Cusick) le personnage-clé, le passeur du show. Il est celui qui guide les disparus : il a des flashforwards avant les autres dès le début de la saison 3, il vit ses flashbacks dès le début de la saison 4 avant que ses amis ne voyagent eux-mêmes dans le temps, et, dans la saison 6, il est doublement présent à l'île et aux flashsideways ce qui lui permet de réunir ses compagnons. Or il ne cesse de répéter : « See you in another life, brother. ", fraternité induisant à la fois une bonne réciprocité mais aussi une compréhension de l'asymétrie, voire de la transcendance supposée par le rapport à autrui, ce vis-à-vis qui m'est une autre vie. Ce qui pourrait être une certaine manière de comprendre la dimension spéciale, spéculaire, de l'esprit selon le philosophe David Hume quand il affirmait que les esprits humains sont les miroirs les uns des autres ${ }^{41}$. Au demeurant, Desmond n'appelle qu'un seul personnage "Sister.», Claire (Emilie DeRavin) dans la première partie du double épisode « Live Together, Die Alone » 
(S02E23). Celle-ci se révélera être effectivement la sœur de Jack et la raison pour laquelle après la destruction du Miroir grâce aux Good Vibrations de Charlie, il décidera de retourner sur l'île pour la tirer des griffer de l'Homme sans nom, simulacre de Locke mais aussi et surtout de Christian. Jack comprend alors qu'il a abandonné sa sœur et du même coup ce qui parasitait l'image de son père, l'empêchait de voir clairement le monde.

Le recours fraternel pourrait donner à The Leftovers, à travers le récit final et récapitulatif de Nora dans "The Book of Nora », un autre sens que celui d'une résilience individuelle dans la diégèse. Il nous autorise à une autre interprétation de la séquence du bouc émissaire du dernier épisode. Nora n'y assume pas tant sa part satanique, ou sa part de fantôme, pour pouvoir se marier avec Kevin en exposant le mystère initial de la série, mais elle assume surtout notre propre regard et en profane la dimension accusatrice sacrée. En regardant le show, c'est nous-mêmes qui faisons disparaître ces $2 \%$, ces autres, cette minorité exclue avec laquelle nous ne voulons pas admettre que nous entretenons une relation de réciprocité fondamentale. Nous lisons The Leftovers et regardons la télévision au prisme de l'Évangile de Kevin en faisant de Nora un bouc émissaire. Cette lens du récit de The Leftovers, sa focale déformante sur les évènements jusque-là raconté, est notre propre plaisir d'identification mêlé de pitié et de crainte, notre catharsis consolatrice au spectacle de ces leftovers. Cependant avec cette révélation parodique, s'ouvre à nous une manière de regarder autrement.

\section{Apocalypse Please}

nce sens "The Book of Nora " laisse poindre l'espoir d'un authentique "montrer télévisuel » où le récit imagé redonnerait une dignité à l'impersonation produite par le médium selon une démarche comparable à celle de Lost. Cet épisode se réfère à Lost comme à une image résiduelle dans ces options de dénomination. Ainsi Nora prend le pseudonyme de Sarah quand elle change de vie. Or Sarah est le nom de la femme de Jack (Matthew Fox) incarnée par Julie Bowen dont on apprend dans l'épisode « Man of Science, Man of Faith " qu'à cause de son accident, son fiancé, Kevin (Anson Mount), décide de la quitter. Ainsi le prénom de la fille de Jill (Margaret Qualley), Penelope, est le nom de la fiancée de Desmond, celui-ci faisant sa première apparition dans le même épisode de Lost pour suggérer à Jack qu'il a réussi à réparer (to fix) Sarah, sans doute par le fait même qu'il le lui ait promis en s'engageant au-delà de ce qu'il estimait être ordinairement sa responsabilité.

Enfin, en termes d'image résiduelle de Lost dans The Leftovers, on peut se demander si le motard qui a gravi l'échelle posée à la fenêtre de la bonne sœur n'est pas interprété par l'acteur Mark Pellegrino qui joue le rôle de Jacob dans la première série. On se prend à rêver d'un caméo assurant au lostie sinon la résurrection, du moins la survivance de l'île dans The Leftovers. Mais non! Ce n'est pas Mark Pellegrino, mais l'acteur Paul Hallett crédité au générique comme The man with leather jacket. Toutefois le fait de le filmer en partie dans le noir en plan en pied puis les traits peu visibles à cause de l'éblouissement du phare de sa moto entretient la confusion. Le fan de David Lynch qu'est Lindelof, ce réalisateur dont on retrouve un machiniste homonyme dans le générique de l'épisode "The Book of Nora », n'aura sans doute pas oublié qu'outre Justin Theroux, Mark Pellegrino apparaît lui aussi dans Mullholland Drive (réal. David Lynch, 2001) tantôt avec les yeux vairons, tantôt non, mais toujours avec son blouson en cuir. Jacob est-il donc là 
sans être là comme l'électrique et alternatif Philip Jeffries dans Twin Peaks? Jacob est mort, vive Jacob? Montrer cette ressemblance d'un acteur qui n'est pas Mark Pellegrino avec celui-ci qui était l'image de Jacob dans Lost, tient d'une définition de l'image comme trace voire résurrection de ce qui est et a passé, dans la mesure où la résurrection rapportée par les Évangiles, à l'instar récit final de Nora à Kevin, est un épisode "loyalement faux» comme l'écrivait Michel Serres ${ }^{42}$. La résurrection n'est pas racontée comme le retour du crucifié ayant strictement la même apparence, elle ne se confond pas avec la répétition à l'identique d'un rassurant déjà-vu et déjà imaginé fantasmatique, si ce n'est dans l'épisode de l'incrédulité de Thomas auquel il est justement reproché de ne pas croire sans voir. Sinon, il est précisé que ses proches ne reconnaissent pas immédiatement le ressuscité, c'est-à-dire qu'ils l'identifient après coup ou sous un autre aspect, un jardinier ou un voyageur. Ainsi l'image comme résurrection serait-elle plutôt une good vibration pour reprendre le titre des Beach Boys grâce auquel Charlie fait traverser le Miroir aux héros de Lost, c'est une bonne onde, une lumière, une manière de voir le quidam, c'est-à-dire n'importe qui autrement et d'en révéler la lumière intérieure, de le rendre spécial, un appel à la grandeur ordinaire ${ }^{43}$.

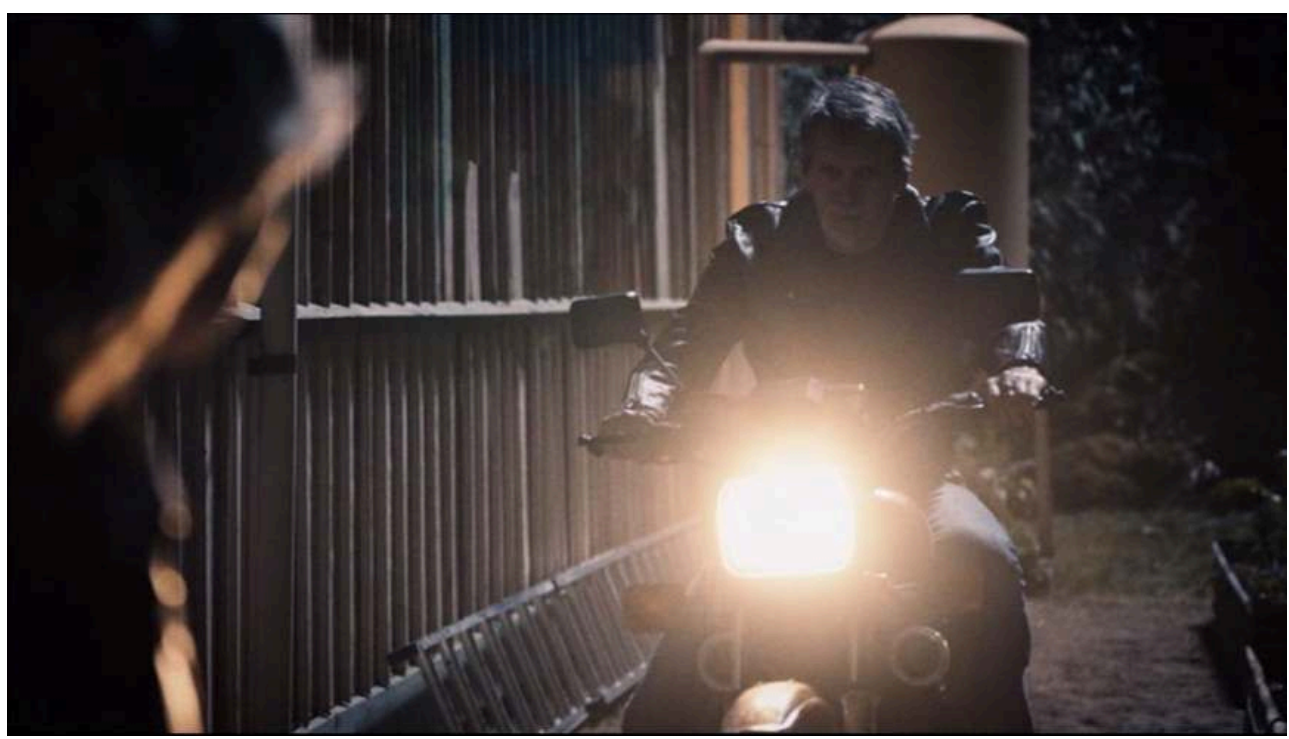

«The Book of Nora » (real. Mimi Leder, 2017)

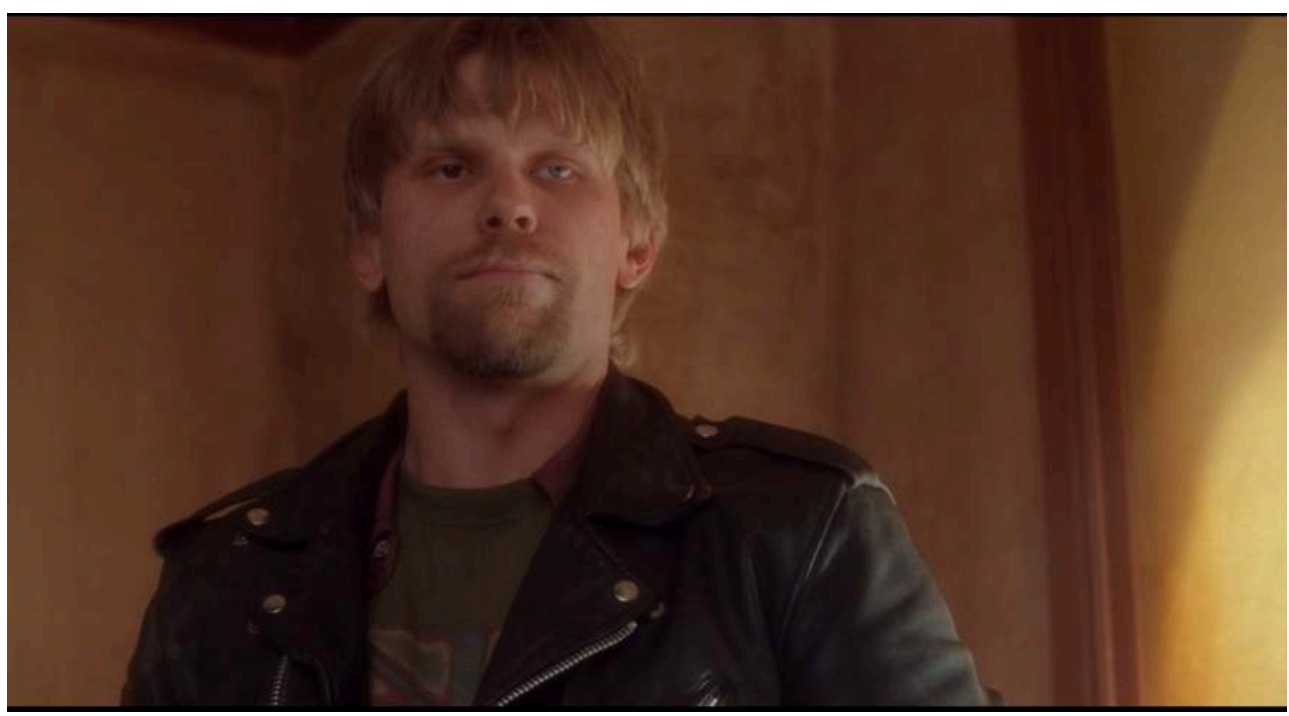

Mulholland Drive (real. David Lynch, 2001) 
56 anodine. Pacôme Thiellement avait déjà montré qu'une bonne part de la première série pouvait se comprendre en regard de Twin Peaks ${ }^{44}$. The Leftovers le précise et l'accentue. Dans la saison 2, deux filles des familles Garvey/Durst et Murphy sont mises en rapport : l'une apparue, Lily, tient et brise sa famille, l'autre disparue, Evangeline, tient et brise aussi sa famille. Même si Nora précise que Lily n'a qu'un « 1 », celui qui a vu Lost peut penser à un clin d'œil à l'actrice Evangeline Lilly, interprète du personnage Kate Austen qui tue l'Homme sans nom. Or l'avant-dernier épisode de The Leftovers, "The most powerfuf man in the world (and his identical twin brother) » se termine sur le morceau de Skeeter Davis, The End of the World, morceau que l'on entend dans un épisode centré sur Kate dans Lost, «What Kate Did » (S02E09), lorsqu'elle annonce à sa mère Diane (Beth Broderick) dans un diner qu'elle l'a débarrassée de son père, ce qui révolte sa mère. Cet épisode prend alors une coloration lynchienne: Kate à présent orpheline, pupille, est hantée par la vision d'un cheval noir (et non pas blanc comme le serait Judy, le blanc de l'œil, de Twin Peaks), elle nous regarde en regardant l'autre en face, les yeux dans les yeux ${ }^{45}$. Lost serait donc une parodie de Twin Peaks, Twin Peaks en télé-évangélisme (comme on parle d'évangélisme de l'Humanisme du XVI siècle, dans les œuvres d'Erasme, More ou Rabelais) ; télé-évangélisme qu'expliciterait The Leftovers par son insistance sur les Books de Kevin et de Nora. Lost bonne nouvelle de Twin Peaks, témoignant que le récit de Lynch était déjà un effort de déparasitage pour sortir de la Black Lodge, profaner l'Improfanable du siècle et son appropriation exclusive des médias, afin de les rendre à l'usage commun dans une parole pure (fire walk with me). Lost rappelle ainsi cette promesse de Twin Peaks de voir le monde clairement et en ce sens Lynch lui-même ne se réfère pas seulement au film Laura (réal. Otto Premminger, 1944). Twin Peaks s'inscrit dans une tradition parodique réagissant au monolinguisme du Canzionere (1374) de Pétrarque, qui lui reprochait d'avoir fait de sa Laura un cadavre. Le geste de cette tradition entend appeler par son nom intime la Bien Aimée, comme le fit Dante pour Béatrice en Paradis dans La Divina Comedia (1321), le nom de Laura aux nues, de Laura nue ${ }^{46}$.

Reste qu'on peut se demander lequel des Kevin revient de l'Apocalypse nucléaire de l'épisode 7 de la saison 3 lorsque retentit The End of the World si, dans The Leftovers, l'île de Lost a totalement disparu à moins que Kevin, comme Kate écoutant The End of the world, regarde enfin en face l'autre, celle qu'il a fui pour ne pas la nommer... en clair, Nora $^{47}$.

AGAMBEN Giorgio, Profanations, Paris, Payot et Rivages, 2006.

HUME David, Treatise of Human Nature, New York, Prometheus Books, 1992.

LINDELOF Damon, entretien, https://collider.com/damon-lindelof-the-leftovers-finaleinterview/\#season-3, consulté le 2 décembre 2019.

_-. https://biiinge.konbini.com/series/interview-damon-lindelof-tom-perrotta-fin-leftovers/, consulté le 2 décembre 2019. 


\section{BIBLIOGRAPHIE}

AUSTIN John Langshaw, Quand dire c'est faire, Paris, Seuil, 1991.

BERGSON Henri, Les Deux sources de la morale et de la religion, Paris, Felix Alcan, 1933.

CAVELL Stanley, The World viewed, Enlarged Edition, Harvard, Havard University Press, 1979 [1971].

DERRIDA Jacques, Donner la mort, Paris, Galilée, 2001.

DULONG Guillaume, «Lost experience : Statut d'Emerson dans la série Lost », TV/Series, Hors séries $\mathrm{n}^{\circ} 1$, 2016, http://journals.openedition.org/tvseries/2207.

__. « La promesse de Lost (À propos de la Saison 2) », TV/Series, Hors séries n¹, 2016, https:// journals.openedition.org/tvseries/1636.

ECO Umberto, Il Nome della Rosa, Milan, Bompiani, 2010.

FRAZER James George, Le Rameau d'Or 3 : Esprits des blés et des bois. Le Bouc émissaire, Paris, Bouquin, 2010.

GIRARD René, La Violence et le Sacré, Paris, Hachette, 1999.

_.. Les Choses cachées depuis la fondation du monde, Paris, Le Livre de Poche, 1983.

__. Le Bouc émissaire, Paris, Le Livre de Poche, 1986.

_-. Achever Clausewitz, Paris, Flammarion, 2011.

HATCHUEL Sarah et THIELLEMENT Pacôme, The Leftovers, le troisième côté du miroir, Paris, Playlist Society, 2019.

HATCHUEL Sarah, Rêves et séries américaines. La fabrique d'autres mondes, Aix-en-Provence, Rouge Profond, 2015.

PEROTTA Tom, The Leftovers, Londres, Fourth Estate, 2011.

SERRES Michel, Relire le Relié, Paris, Le Pommier, 2019.

THIELLEMENT Pacôme, Les Mêmes Yeux que Lost, Paris, Léo Scheer, 2012.

TODOROV Tzvetan, Introduction à la Littérature fantastique, Paris, Seuil, 1976.

\section{NOTES}

1. Georges Brassens, Le 22 Septembre.

2. Giorgio Agamben, Profanations, Paris, Payot et Rivages, 2006, p. 58-59.

3. Tzvetan Todorov, Introduction à la littérature fantastique, Paris, Seuil, 1976, p. 28-45.

4. C'est le récurrent : « Don't mistake coincidence for fate. » émaillant les dialogues de Lost et, mutatis mutandis, les ruminations du Dr Cuarto (Diego Wallraff) de l'épisode «Lens» (S0206) de The Leftovers. Ce savant tente de distinguer la causalité scientifique rationnelle d'une corrélation mnésique et imaginaire dont il dit que même Denzinger serait capable - Denziger étant le patronyme d'un sénateur dans la première saison dont nous comprenons, alors, qu'il peut aussi être une allusion homonymique à un théologien allemand catholique du XIX ${ }^{\mathrm{e}}$ siècle.

5. Henri Bergson, Les Deux Sources de la morale et de la religion, Paris, Felix Alcan, 1933, p.317-328. René Girard, Achever Clausewitz, Paris, Flammarion, 2011, p. 149. 
6. https://collider.com/damon-lindelof-the-leftovers-finale-interview/\#season-3

7. Giorgio Agamben, Profanations, Paris, Payot et Rivages, 2006, p. 95.

8. Hatchuel et Thiellement, op. cit., p. 24-26.

9. Ibid., p. 41-48. Sur « The Book of Nora », est mobilisée une référence à La Nuit des Rois comédie dite « à fin forcée " pour en montrer la dimension particulièrement invraisemblable en regard de la cohérence du reste du récit (p. 134-137).

10. Agamben, op. cit., p. 72.

11. Giorgio Agamben, op. cit., p. 76.

12. Guillaume Dulong, « La promesse de Lost (À propos de la Saison 2) »,TV/Series, Hors séries n¹, 2016, http://tvseries.revue.org/1636.

13. Assez ironiquement la plupart des reproches de «vol » de vie ou de temps adressés par les détracteurs de la série aux showrunners sur les réseaux sociaux lors de la diffusion du dernier épisode en 2010, reprirent mutatis mutandis les répliques du personnage de John Locke enrageant de dépit en découvrant la Perle dans l'épisode de 2006, «?» (S02E21). Or ce personnage est celui dont la série entérine la chute jusqu’à devenir la manifestation du monstre de fumée à partir la moitié de la saison 5 puis jusqu'à la fin.

14. L'une des quelques occurrences significatives de l'expression left over dans Lost se trouve dans l'épisode «A tale of two cities » (S01E03) lorsque Jack demande à Juliet (Elizabeth Mitchell) si les Autres sont ceux qui restent («So you people are just whatever's left over of them?») de la Dharma Initiative où l'expression désigne un groupe antagoniste à celui des disparus pour les disparus.

15. Le titre du roman de Golding reprend l'un des titres de Belzébuth, donc la communauté de la queue de l'appareil se fait sous le signe d'un ange, d'une médiation déchue.

16. Sur la grandeur commune et l'ambivalence du statut du special guest dans les séries télévisées on peut se reporter au problème de la confiance en soi chez Emerson mis en rapport à l' impersonation télévisuelle chez Cavell et à l'éthique de l'accueil dans Mission Impossible et Lost. Guillaume Dulong, «Lost experience : Statut d'Emerson dans la série Lost », TV/Series, Hors séries $\mathrm{n}^{\circ} 1,2016$, http://journals.openedition.org/tvseries/2207.

17. John Langshaw Austin, Quand dire c'est faire, Paris, Seuil, 1991.

18. Agamben, op. cit., p. 100-107.

19. Carrie est le prénom de l'hérö̈ne souffre-douleur du roman de Stephen King, qui est le livre préféré de Juliet Burke (Elizabeth Mitchell) dans Lost et sur la critique duquel s'ouvre sa troisième saison.

20. Le premier épisode de la série, "Pilot», déconstruit cette communion citoyenne commémorative autour de la souffrance de Nora, déconstruction culminant dans l'intervention des Guilty Remnant qui se feront molester par la police au son de la sonate ${ }^{\circ} 20$ de Schubert.

21. Le personnage de Kevin est maire de la ville dans le roman de Perotta.

22. Les rituels grecs et d'Asie mineure du pharmakos et du diaspragmos dans leur dimension littéralement et étymologiquement tragiques peuvent être considérés analogiquement comme une autre source de l'expression moderne. Pour la tradition chrétienne, ce rituel peut être entendu comme préfigurant le sacrifice unique ou parfait de l'agneau sans péché que serait le Christ.

23. Tom Perotta, The Leftovers, Londres, Fourth Estate, 2011, p. 106.

24. James Georges Frazer, Le Rameau d'Or. Esprits des Blés et des Bois. Le Bouc émissaire, Paris, Robert Laffont, 1983.

25. René Girard, La Violence et le Sacré, Paris, Hachette, 1998.

26. Guillaume Dulong, « La promesse de Lost (À propos de la Saison 2) », TV/Series, Hors séries n¹, 2016, http://tvseries.revue.org/1636. Ce dilemme moral travaille l'œuvre de Spielberg, autre source d'inspiration de Lindelof. The Schindler's List (1993) s'offre comme une réflexion sur une phrase que l'on retrouve dans le Talmud et le Coran : «Celui qui sauve une vie sauve l'humanité toute entière. " et qui insiste sur la nécessaire disproportion conditionnant ce précepte dans Saving 
Private Ryan (1998) où une compagnie va mettre sa vie en jeu pour ramener un simple soldat à sa mère qui a perdu tous ses fils.

27. René Girard, Le Bouc émissaire, Paris, Le Livre de Poche, 1986, p. 235.

28. Il ne s'en tient donc pas non plus à l'analyse produite par Frazer en 1913, puisqu'il déplore son dédain pour une pensée magique réduite à un raisonnement inexact et infantile. Ce dédain témoigne d'une ignorance des mécanismes psychosociaux conditionnant le sentiment d'efficacité du rituel sacrificiel, efficacité reposant sur la méconnaissance.

29. René Girard, La Violence et le Sacré, Paris, Hachette, 1998, p. 212-248.

30. D'ailleurs dans l'épisode «The Economist » (S04E03), Sayid (Naveen Andrews) découvre sur le corps de Naomie un bracelet d'argent où est gravé : "N. I'll always be with you. R.G.» R.G.? Remnant Guilty ? Hergé ? René Girard ? On peut se poser la question dans la mesure où l'intitulé même de cet épisode, "The Economist ", interpelle : l'économiste est un individu que Sayid aux ordres de Ben (Michael Emerson) doit tuer mais que l'on ne verra jamais. L'acception prosaïque du terme "économie » liée à la production et aux échanges de biens peut se comprendre comme la sécularisation de sa signification théologique et iconologique au moins pour les Chrétiens. Dans un sens théologique, l'économie est la manière dont Dieu se révèlerait ici-bas et donc une manière de comprendre l'histoire humaine à l'aune de sa dimension sotériologique. C'est aussi corrélativement l'ensemble des procédés et codes iconographiques à suivre afin de rendre visible cette intervention sacrée et invisible, particulièrement par, avec et dans le prototype, l'incarnation christique, à partir de laquelle serait autorisée la représentation des saints et prophètes. La question : «Qui est l'économiste de Lost ? " prend alors un autre sens. Est-ce Jacob ? Son double? Benjamin? Hugo? Lindelof? Cuse? Nous? Toujours est-il que cette double définition de l'économie à la fois profane et sacrée s'accorde avec l'anthropologie de Girard.

31. René Girard, Les Choses cachées depuis la fondation du monde, Paris, Le Livre de Poche, 1983, p. 356-376.

32. Jacques Derrida, Donner la mort, Paris, Galilée, 2001. Guillaume Dulong, « Lost experience : Statut d'Emerson dans la série Lost », TV/Series, Hors séries n¹, 2016, http://journals.openedition.org/ tvseries/2207.

33. Michel Serres, Relire le relié, Paris, Le Pommier, 2019, p. 238.

34. " Hic sunt leones » écrivait Umberto Eco dans Il Nome della Rosa, quand les héros se retrouvait devant une fresque de l'Apocalypse dans le labyrinthe de la bibliothèque de l'abbaye. Or une fresque apocalyptique ouvrait semblablement le générique de la première saison du labyrinthe Leftovers. Umberto Eco, Il Nome della Rosa, Milan, Bompiani, 2010, p. 313-329.

35. Giorgio Agamben, op. cit., p. 75.

36. Sarah Hatchuel et Pacôme Thiellement, The Leftovers, le troisième côté du miroir, Paris, Playlist Society, 2019, p. 132-133.

37. https://collider.com/damon-lindelof-the-leftovers-finale-interview/\#season-3

38. Agamben, op. cit., p. 42-43 et p. 57-58.

39. René Girard, Le Bouc émissaire, Paris, Livre de Poche, 1986, p. 174-175.

40. L'allégorie elle-même est une manière de tenir à distance, de contenir, la violence originelle que mime le sacrifice et verbalise le récit. Le sens allégorique est donc une manière de détourner la violence tout en la conservant, à l'instar de la fonction écran de l'image fantasmatique qui contient des pulsions érotiques polymorphes dans la psychologie freudienne. Celle-ci s'avoue le refoulement mais pas le refoulé : elle est violence renversée contre elle-même pour en différer les affres et, par là-même, se voile. La mécanique du désir, sa projection sur un substitut, et le différé qu'opère sa violence cathartique demeurent méconnus.

41. David Hume, Treatise of Human Nature, New York, Prometheus Books, 1992, p. 365.

42. Michel Serres, op. cit., p. 182-188.

43. Guillaume Dulong, "Lost experience: Statut d'Emerson dans la série Lost », TV/Series, Hors séries $n^{\circ} 1,2016$, http://journals.openedition.org/tvseries/2207. 
44. Pacôme Thiellement, Les Mêmes Yeux que Lost, Paris, Léo Scheer, p.100-101.

45. Sarah Hatchuel montre cette proximité des personnages de Laura Palmer (Sheryl Lee) dans Twin Peaks et de Kate Austen dans Lost en pointant l'opposition des chevaux. Le cheval blanc conditionnant l'enfer de la Red Room comme matrice d'un monde médiatique mensonger, le cheval noir est donc a contrario le moyen d'en sortir. Sarah Hatchuel, Rêves et séries américaines. La fabrique d'autres mondes, Aix-en-Provence, Rouge Profond, 2015, p. 249. En réutilisant la chanson The End of the World, Lindelof relie The Leftovers au projet de Lost en l'explicitant dans les deux derniers épisodes de la dernière saison. Ainsi fait-il sortir Kevin de l'hysteria de son autre monde offrant une voie de salut au show télévisé par le livre de Nora.

46. Pour reprendre le titre d'un film de Niccolo Ferrari de 1961 et la distinction entre la nudité profane d'inspiration lévinassienne et l'exposition séculaire et pornographique proposée par Agamben, op. cit. p. 55-56 et p. 117-122.

47. En ce sens The Leftovers offre une solution à ce que Tomorrowland (réal. Brad Bird, 2015) du propre aveu de Lindelof pouvait avoir de frustrant. Sans doute dans son principe de composition et d'écriture ce film ne permettait-il pas au spectateur d'engager son propre regard en comprenant le récit audiovisuel comme la relation de Frank (Georges Clooney) et Cassie (Britt Robertson), faisant du film lui-même une projection du Monitor.

\section{RÉSUMÉS}

Cet article analyse le dernier épisode de The Leftovers, "The Book of Nora " (S03E08), en son opposition avec l'épisode "The Book of Kevin" (S03E01) comme légendes interprétatives concurrentes de la série en se focalisant sur le thème du bouc émissaire et son traitement audiovisuel ramené à la persona spéciale de Nora (Carrie Coon). Ce faisant, nous nous demandons ce que signifie l'accusation proférée à l'encontre de celle-ci dans l'épisode «Lens " (S02E06) d'être Azraël et en quoi The Leftovers est apocalyptique. Il ne s'agit pas uniquement de désigner ce personnage responsable de l'incident par lequel s'origine l'histoire, elle est celle qui répond de la vraie version du récit mais aussi nous dévoile notre regard accusateur sur ce dernier. Ainsi en télescopant les définitions de la profanation parodique de Giorgio Agamben et les études de René Girard sur le phénomène socioculturel de bouc émissaire, prétendons-nous dégager la montée aux extrêmes des tensions, la double frénésie traversant The Leftovers intérieurement, mais aussi dans son rapport à la série précédente de Damon Lindelof, Lost. Peut-on espérer le retour de l'île de Lost dans The Leftovers?

This essay analyses The Leftovers's last episode, "The Book of Nora" (S03E08), in its opposition with the episode "The Book of Kevin" (S03E01), as rival interpretative legends focusing on the scapegoat theme and its audiovisual processing in reference to the special persona of Nora (Carrie Coon). Meanwhile we interrogate the meaning of the accusation of being Azraël uttered against her in the episode "Lens" (S02E06) and what makes The Leftovers an apocalyptic show. Far from designating Nora as responsible for the original incident, we see her as being the one who represents the true version of the narrative and reveals to us our own accusing look on it. Then, associating the Giorgio Agamben's parodical profanation definition with René Girard's studies on the sociocultural phenomenon of the scapegoat, we'll attempt to reveal the rising tensions and double frenzy throughout The Leftovers, inside the fiction but also in regard of Damon Lindelof's previous television show, Lost. Can we expect a return of Lost's Island in The Leftovers? 


\section{INDEX}

Keywords : apocalypse, scapegoat, special, profanation, Lindelof Damon, Girard René, Agamben Giorgio, Leftovers (The), Lost, parody, tele-evangelism

Mots-clés : apocalypse, bouc émissaire, spécial, profanation, Lindelof Damon, Girard René, Agamben Giorgio, Leftovers (The), Lost, parodie, télé-évangélisme

\section{AUTEUR}

\section{GUILLAUME DULONG}

Guillaume Dulong, agrégé de philosophie et docteur en études cinématographiques, est professeur de philosophie et de cinéma en lycée, ainsi que chargé de cours en cinéma à l'université Michel de Montaigne Bordeaux. S'inspirant des théories d'André Gaudreault et Tom Gunning, son travail de thèse a porté sur le genre cinématographique de l'imaginaire et l'influence des technologies numériques sur les modes de narration contemporains de fantasy. Guillaume Dulong, who holds an 'agrégation' in philosophy and a PhD in film studies, is a professor of philosophy and cinema in high school; he also teaches cinema at the University of Bordeaux Michel de Montaigne. Inspired by the theories of André Gaudreault and of Tom Gunning, his PhD thesis explored the filmic genre of fantasy and the influence of digital technologies on contemporary narration. 